\title{
Pädagogisches Wissen versus fachdidaktisches Wissen?
}

\section{Struktur des professionellen Wissens bei angehenden Deutsch-, Englisch- und Mathematiklehrkräften im Studium}

\author{
Johannes König (D) · Jörg Doll • Nils Buchholtz • Sabrina Förster • \\ Kai Kaspar • Anna-Maria Rühl • Sarah Strauß • Albert Bremerich-Vos • \\ Ilka Fladung • Gabriele Kaiser 1
}

Online publiziert: 10. Juli 2017

(C) Der/die Autor(en) 2017. Dieser Artikel ist eine Open-Access-Publikation.

Zusammenfassung Pädagogisches und fachdidaktisches Wissen gelten als zentrale kognitive Elemente professioneller Kompetenz von Lehrkräften. Der Erwerb entsprechenden Wissens soll daher möglichst schon im Lehramtsstudium erfolgen. Wie allerdings pädagogisches Wissen und fachdidaktisches Wissen zueinander in Verbindung stehen bzw. voneinander abgegrenzt werden können, ist konzeptionell wie empirisch noch weitgehend ungeklärt. Vor diesem Hintergrund bearbeitet der vorliegende Beitrag mithilfe standardisierter Wissenstests und auf der Basis von 889

Prof. Dr. J. König $(\bowtie) \cdot$ Dr. S. Strauß

Empirische Schulforschung, Quantitative Methoden, Department Erziehungs- und

Sozialwissenschaften, Universität zu Köln, Gronewaldstr. 2, 50931 Köln, Deutschland

E-Mail: johannes.koenig@uni-koeln.de

Dr. S. Strauß

E-Mail: sarah.strauss@uni-koeln.de

Prof. Dr. J. Doll

Projektevaluation ProfaLe, Qualitätsoffensive Lehrerbildung Fakultät für Erziehungswissenschaft, Universität Hamburg, Max-Brauer-Allee 58/60, 22765 Hamburg, Deutschland

E-Mail: joerg.doll@uni-hamburg.de

Prof. Dr. N. Buchholtz

Department of Teacher Education and School Research Faculty of Educational Science, University of Oslo, Postbox 1099 Blindern, 0317 Oslo, Norwegen

E-Mail: n.f.buchholtz@ils.uio.no

S. Förster · A.-M. Rühl

Qualitätssicherung ZuS, Qualitätsoffensive Lehrerbildung, Empirische Schulforschung, Quantitative Methoden, Department Erziehungs- und Sozialwissenschaften, Universität zu Köln, Gronewaldstr. 2a, 50931 Köln, Deutschland

S. Förster

E-Mail: sabrina.foerster@uni-koeln.de

A.-M. Rühl

E-Mail: anna-maria.ruehl@uni-koeln.de 
Lehramtsstudierenden der Fächer Deutsch, Englisch und Mathematik von zwei Volluniversitäten die beiden folgenden Fragen: (1) Lässt sich pädagogisches Wissen von Studierenden empirisch von ihrem deutsch-, englisch- und mathematikdidaktischen Wissen trennen? (2) Bestehen Unterschiede im fachdidaktischen Wissen in Abhängigkeit von ihrem Ausbildungsstadium (Bachelor vs. Master) und können diese zusätzlich durch spezifische Lerngelegenheiten in der Fachdidaktik erklärt werden (im Gegensatz zum pädagogischen Wissen)? Die Ergebnisse zeigen zum einen, dass sich pädagogisches Wissen und fachdidaktisches Wissen wie erwartet empirisch trennen lassen. Dies gilt für jede der drei Domänen (Konstruktvalidität), wobei pädagogisches Wissen mit mathematikdidaktischem niedriger korreliert als mit deutsch- bzw. englischdidaktischem Wissen (bei Kontrolle von Hintergrund- und Eingangsmerkmalen der Studierenden und Merkmalen ihrer Lerngelegenheiten). Zum anderen indizieren Kohorten-Vergleiche von Bachelor- und Master-Studierenden ein besseres Abschneiden letzterer in allen Domänen für das fachdidaktische und pädagogische Wissen (curriculare Validität). Deutschdidaktisches und englischdidaktisches Wissen lassen sich darüber hinaus stärker als mathematikdidaktisches Wissen durch fachdidaktische Lerngelegenheiten erklären (konvergente Validität) und vom pädagogischen Wissen abgrenzen (diskriminante Validität). Implikationen für aktuell stattfindende Maßnahmen der Qualitätssicherung in der universitären Lehrerbildung werden diskutiert.

Schlüsselwörter Fachdidaktisches Wissen · Kompetenz · Lehrerbildung · Lehramtsstudierende · Lerngelegenheiten · Pädagogisches Wissen

Jun.-Prof. Dr. Dr. habil. K. Kaspar

Sozial- und Medienpsychologie, Department Psychologie, Universität zu Köln,

Richard-Strauss-Str. 2, 50931 Köln, Deutschland

E-Mail: kkaspar@uni-koeln.de

Prof. Dr. A. Bremerich-Vos

Germanistische Linguistik und Sprachdidaktik, Universität Duisburg-Essen, Berliner Platz

6-8, 45127 Essen, Deutschland

E-Mail: Albert.Bremerich-Vos@uni-due.de

\section{Fladung}

Fakultät für Geisteswissenschaften, Germanistische Linguistik und Sprachdidaktik, Universität Duisburg-Essen, Berliner Platz 6-8, 45127 Essen, Deutschland

E-Mail: ilka.fladung@uni-due.de

Prof. Dr. G. Kaiser

Fakultät für Erziehungswissenschaft, Didaktik der Mathematik, Universität Hamburg,

Von-Melle-Park 8, 20146 Hamburg, Deutschland

E-Mail: gabriele.kaiser@uni-hamburg.de

Learning Sciences Institute, Australian Catholic University, Brisbane, Australien 


\title{
General pedagogical knowledge versus pedagogical content knowledge?
}

The structure of professional knowledge in pre-service teachers of German, English, and Mathematics at university

\begin{abstract}
General pedagogical knowledge (GPK) and pedagogical content knowledge (PCK) are central cognitive elements in the professional competence of teachers. Thus initial teacher education aims at the acquisition of this relevant knowledge. However, the question how GPK and PCK are related to each other or can be separated from each other has not yet been answered, neither conceptually nor empirically. Against this background, this article focuses on two research questions on the basis of standardized knowledge tests administered to 889 pre-service teachers of German, English and Mathematics from two large universities: (1) Can GPK empirically be separated from PCK of German, English, and Mathematics? (2) Are there differences in pre-service teachers' PCK test scores depending on their stage of training (Bachelor versus Master) and can PCK test scores additionally be explained by specific learning opportunities in the area of PCK (when compared with GPK test scores)? On the one hand, findings show that GPK and PCK can empirically be separated. This is true for all three domains (construct validity), whereby the correlation between GPK and Mathematics PCK is lower than the correlations between GPK and German PCK and English PCK (when controlled for background and entry characteristics of students and their learning opportunities). On the other hand, a comparison between cohorts of Bachelor and Master student teachers provides evidence that the more advanced Master students outperform the Bachelor students in all knowledge tests (curricular validity). In addition, German PCK and English PCK (better than Mathematics PCK) can be explained by subject-specific learning opportunities (convergent validity) and be demarcated from GPK (discriminant validity). Implications for current initiatives of quality assurance in initial teacher education are discussed.
\end{abstract}

Keywords Competence - General pedagogical knowledge - Opportunities to learn · Pedagogical content knowledge $\cdot$ Pre-service teachers $\cdot$ Teacher education

\section{Einleitung}

Die universitäre Lehramtsausbildung in Deutschland gliedert sich üblicherweise in fachwissenschaftliche, fachdidaktische und pädagogische (bzw. bildungswissenschaftliche ${ }^{1}$ ) Studienanteile. Während das Studium eines Faches, insbesondere bei Sekundarstufenlehrkräften, disziplinorientiert und weitgehend unabhängig vom Berufsziel Lehrer bzw. Lehrerin konzipiert ist, stehen Fachdidaktik und Pädagogik/ Bildungswissenschaften deutlich stärker in einem funktionalen Zusammenhang mit dem Lehrerberuf, insofern von ihnen erwartet wird, dass sie die zukünfti-

\footnotetext{
${ }^{1}$ Wir beziehen uns hier auf die von der KMK (2004/2014) getroffene Bezeichnung. Das Studienelement „Bildungswissenschaften“ umfasst nicht allein Anteile in Pädagogik oder Erziehungswissenschaft, sondern auch in Psychologie, Soziologie, Philosophie, Politikwissenschaft (vgl. detailliert Terhart 2012).
} 
gen Lehrkräfte auf die Bewältigung beruflicher Anforderungen vorbereiten sollen (KMK 2004/2014, 2008). Fachdidaktik und Pädagogik gelten gleichermaßen als Bezugsdisziplinen der Profession: Sie unterstützen die schulpraktische Ausbildung während des Studiums, vor allem aber finden sie ihre Fortsetzung in der praktischen Ausbildungsphase, dem Referendariat bzw. Vorbereitungsdienst. Der Aufbau von fachdidaktischem und pädagogischem Wissen ${ }^{2}$, an das die zweite Phase effektiv anschließen kann, ist bereits Ziel eines universitären Lehramtsstudiums.

Aktuell schenken Reformdiskurse zur ersten Phase der Lehrerbildung den Fachdidaktiken besondere Aufmerksamkeit, wird bei ihnen doch ein beträchtliches Potenzial zur Optimierung der Lehramtsausbildung insgesamt vermutet (z. B. Expertenkommission 2007). ${ }^{3}$ Beispielsweise manifestieren sich die in der Pädagogik bzw. den Bildungswissenschaften behandelten didaktischen Fragen häufig erst über die zu unterrichtenden Fachinhalte des schulischen Curriculums und müssen somit von Lehrkräften domänenspezifisch bearbeitet werden (u. a. Baumert et al. 2010), denn das im Unterricht anzuregende Lernen der Schülerinnen und Schüler geschieht vor allem inhaltsspezifisch (u. a. Weinert 1994). Der Erwerb fachdidaktischen Wissens von Lehrpersonen wird daher durch domänenspezifische Lerngelegenheiten unterstützt, die speziell in der Verantwortung fachdidaktischer Lehre liegen und nicht von den Bildungswissenschaften übernommen werden können. Gleichwohl wird die Bedeutung von pädagogischem Wissen von Lehrpersonen neben ihrem fachlichen Wissen darin gesehen, dass fachdidaktisches Wissens eine Art „Amalgam“ von pädagogischem und fachlichem Wissen sei (Shulman 1987, S. 8; vgl. auch MorineDershimer und Kent 1999). So ist es nachvollziehbar, dass Reforminitiativen aktuell eine stärkere Zusammenarbeit der Fachdidaktiken unterschiedlicher Fächer mit den Bildungswissenschaften in der Lehrerausbildung erwarten (z. B. BMBF 2014).

Fachdidaktisches und pädagogisches Wissen bilden zusammen mit dem Fachwissen eine Trias, die in der Literatur auch als das ,professionelle Wissen“ (Bromme 1992) bezeichnet und in einschlägigen Kompetenzmodellen als kognitiver Bereich verortet wird (Baumert und Kunter 2006; Blömeke et al. 2010). Wie allerdings fachdidaktisches und pädagogisches Wissen bei Lehrkräften miteinander in Verbindung stehen bzw. voneinander abgegrenzt werden können, ist nach wie vor konzeptionell unklar (z. B. Blömeke et al. 2007), vor allem aber eine weitgehend unbearbeitete Frage der empirischen Bildungsforschung. Die standardisierte Erfassung von professionellem Wissen der Lehrerinnen und Lehrer ist in den vergangenen Jahren deutlich vorangeschritten (Baumert 2016). Während einige empirische Studien die Beziehung zwischen Fachwissen und fachdidaktischem Wissen analysieren (u.a. Baumert et al. 2010; Blömeke et al. 2011; Blömeke und Delaney 2012; Jansing et al. 2013; Leung et al. 2016), wurde der Analyse der Beziehung zwischen pädagogischem und fachdidaktischem Wissen bislang nur wenig Beachtung geschenkt (vgl.

\footnotetext{
${ }^{2}$ Mit der Verwendung des Begriffs ,pädagogisches Wissen“ folgen wir zunächst einer sprachlichen Darstellung, wie sie z. B. im Review von Voss et al. (2015) zu finden ist, auch wenn dabei - analog zum Begriffspaar „Pädagogik“ und „Bildungswissenschaften“ (s. Endnote 1) - nicht nur pädagogisches Wissen, sondern möglicherweise auch pädagogisch-psychologisches Wissen, bildungssoziologisches Wissen usw. angesprochen sein kann.

${ }^{3}$ Diese Frage ist allerdings nicht neu, sondern bildet seit Jahren eine Reformfrage der Lehrerbildung, und zwar nicht nur in Deutschland (vgl. bereits Reusser 1991).
} 
Voss et al. 2015), insbesondere unter der Berücksichtigung verschiedener Domänen (Blömeke et al. 2011; König et al. 2011; Marx et al. 2014; Lenske et al. 2015).

An dieser Problemlage setzt der vorliegende Beitrag an. Vorgestellt werden Konzepte zur Erfassung von fachdidaktischem Wissen von angehenden Deutsch-, Englisch- und Mathematiklehrkräften der Sekundarstufe, verbunden mit ihrem pädagogischen Wissen. ${ }^{4}$ Teils wird auf bekannte, teils auf neuere Testinstrumente zurückgegriffen. Auf Basis von Untersuchungen mit Lehramtsstudierenden in der Bachelorund Masterphase von zwei Volluniversitäten in Deutschland wird die Unterscheidung des professionellen Wissens in pädagogisches und fachdidaktisches Wissen nach Fächern strukturell geprüft und Lernzuwächse werden über Kohorten-Vergleiche quasi-längsschnittlich abgeschätzt. Ziel dieser Strukturanalysen ist es, Aussagen zur Konstruktvalidität der verwendeten Tests zu treffen, insbesondere anhand der Frage, ob fachdidaktisches Wissen von pädagogischem Wissen empirisch getrennt werden kann. Die Frage nach Lernzuwächsen bei angehenden Lehrkräften zielt hingegen darauf, curriculare Validität der Tests zu belegen. Darüber hinaus werden Indikatoren fachspezifischer Lerngelegenheiten als Prädiktoren einbezogen, mit denen Leistungen in fachdidaktischen Wissenstestungen regressionsanalytisch erklärt sowie von Leistungen im pädagogischen Wissenstest abgegrenzt werden sollen. Die Ergebnisse werden als Hinweise auf die Wirksamkeit der universitären Lehramtsausbildung diskutiert sowie in aktuelle Entwicklungen der Lehrerbildungsreform wie die „Qualitätsoffensive Lehrerbildung“ (BMBF 2014) eingeordnet.

\section{Forschung zum Wissen angehender Lehrkräfte}

\subsection{Wissen als Teil der professionellen Kompetenz von Lehrkräften}

In den vergangenen Jahren hat die Forschung zum Wissen angehender und berufstätiger Lehrkräfte stark zugenommen. Insbesondere Arbeiten, die sich um eine standardisierte Erfassung bemühen, beziehen sich auf Überlegungen zum Lehrerwissen (u. a. Shulman 1987; Grossman 1990) und zum „professionellen Wissen“ von Lehrkräften im Kontext des Expertise-Paradigmas (Bromme 1992; Hogan et al. 2003). Das professionelle Wissen wiederum wird in aktuellen Modellen professioneller Kompetenz als kognitive Komponente verortet (Baumert und Kunter 2006; zu aktuellen Entwicklungen vgl. auch Blömeke et al. 2015).

Die in diesen Kompetenzmodellen zur Bewältigung berufsbezogener Anforderungen - insbesondere des Unterrichtens - notwendigen kognitiven Leistungsdispositionen werden analytisch weiter ausdifferenziert, und zwar in fachliches, fachdidaktisches und pädagogisches Wissen (Shulman 1987; Bromme 1992; Baumert und Kunter 2006; Blömeke et al. 2010), auch bekannt als content knowledge (CK), pedagogical content knowledge (PCK) und general pedagogical knowledge (GPK).

\footnotetext{
${ }^{4}$ Deutsch und Mathematik sind Kernfächer, in den meisten Bundesländern gilt dies auch für Englisch. Der Fokus auf Englisch als Fremdsprache begründet sich ferner durch die Bedeutung von Englisch als lingua franca (House 2003; Jenkins 2013), als die einflussreichste Fremdsprache weltweit (Weber 1997) und als die am häufigsten unterrichtete Fremdsprache in Schulen Europas (Eurostat 2012).
} 
Diese Trias korrespondiert mit jenen wissenschaftlichen Disziplinen (Fachdisziplin, Fachdidaktik, Pädagogik bzw. Bildungswissenschaft), die typische Komponenten der universitären Lehrerausbildung darstellen (KMK 2004, 2014, 2008). Gleichzeitig handelt es sich um eine analytische Differenzierung von Wissenskomponenten, über die Lehrpersonen bei der Bewältigung berufsbezogener Anforderungen, speziell beim Unterrichten, verfügen müssen (Shulman 1987; Bromme 2001; Baumert und Kunter 2006; Bremerich-Vos und Dämmer 2013). In kompetenzorientierten Ansätzen (Blömeke et al. 2015) werden Kompetenzen in Form von Lehrerwissen als grundsätzlich erlernbare Fähigkeiten betrachtet, die zeitlich und situationsübergreifend als relativ stabile Dispositionen gelten und so operationalisiert werden können, dass sie mithilfe testdiagnostischer Verfahren einer Erfassung zugänglich sind.

\subsection{Inhaltliche Differenzierungen in pädagogisches und fachdidaktisches Wissen}

\subsubsection{Pädagogisches Wissen}

Mit Shulman (1987, S. 8) kann pädagogisches Wissen zunächst verstanden werden als ,knowledge, with special reference to those broad principles and strategies of classroom management and organization that appear to transcend subject matter". Hierbei ist es von Bedeutung, dass Shulman (1987) wie auch weitere Autorinnen und Autoren (vgl. Baumert und Kunter 2006; Baumert et al. 2010; Tatto et al. 2012) dieses Wissen von verschiedenen anderen Komponenten des professionellen Wissens konzeptionell abgrenzen, vor allem vom fachbezogenen Wissen.

Ansätze zur Definition von pädagogischem Wissen und seiner inneren Struktur enthalten unterschiedliche Differenzierungen. Inhaltlich betrachtet werden zum Beispiel in einer von Baumert und Kunter (2006, S. 485) gegebenen Übersicht vier Themen (konzeptuelles bildungswissenschaftliches Grundlagenwissen, allgemeindidaktisches Konzeptions- und Planungswissen, Unterrichtsführung und Orchestrierung von Lerngelegenheiten, fachübergreifende Prinzipien des Diagnostizierens, Prüfens und Bewertens) mit Unteraspekten gelistet, die zeigen, dass pädagogisches Wissen mit Bezug auf Anforderungen des Unterrichtens (z. B. effektive Klassenführung) beschrieben werden kann, jedoch auch über unterrichtliche Inhalte hinausgeht (z. B. historische Grundlagen von Schule; vgl. auch Terhart 1993; Oser 2014).

In jüngster Zeit erschienene Überblicksarbeiten fassen vorhandene Ansätze mit Blick auf die Kernaufgabe des Unterrichtens zusammen und identifizieren inhaltliche Kernbereiche, die über die vorhandenen Studien hinweg auffindbar sind: Auf Basis von Forschung im deutschsprachigen Raum gelangen Voss et al. (2015) dabei in inhaltlicher Hinsicht zu den Bereichen „Lernen und Lernende“, „Umgang mit der Klasse als komplexem sozialen Gefüge“" und „methodisches Repertoire“. Im Rahmen eines systematischen Reviews internationaler Literatur identifiziert König (2014) die drei Dimensionen instructional process, student learning, und assessment. Bei einer Gegenüberstellung der beiden Übersichten (Abb. 1) wird deutlich, dass sie im Kern große inhaltliche Übereinstimmungen aufweisen. Lediglich der Bereich instructional process ist bei König (2014) etwas breiter gefasst und deckt damit bereits einen Teil des „methodischen Repertoires“ von Voss et al. (2015) ab, während dort Diagnostik 


\begin{tabular}{|c|c|}
\hline Voss et al. (2015, S. 194) & König $(2014$, S. 49) \\
\hline $\begin{array}{l}\text { Lernen und Lernende: } \\
\text { - Lernprozesse (lern-, motivations- und } \\
\text { emotionspsychologisches Wissen) } \\
\text { - Unterschiede in den Voraussetzungen der } \\
\text { Lernenden (Heterogenität) } \\
\text { - Altersstufen und Lernbiographien } \\
\text { (entwicklungspsychologisches Wissen) }\end{array}$ & $\begin{array}{l}\text { student learning } \\
\text { (including their cognitive, motivational, } \\
\text { emotional individual dispositions; their learning } \\
\text { processes and development; their learning as a } \\
\text { group taking therefore into account student } \\
\text { heterogeneity and adaptive teaching strategies), }\end{array}$ \\
\hline $\begin{array}{l}\text { Umgang mit der Klasse als komplexem sozialen } \\
\text { Gefüge: } \\
\text { - Klassenführung/Strukturierung der } \\
\text { Klassenprozesse } \\
\text { - Interaktion/Kommunikation und soziale } \\
\text { Konflikte }\end{array}$ & \multirow[t]{2}{*}{$\begin{array}{l}\text { instructional process } \\
\text { (including teaching methods, didactics, } \\
\text { structuring a lesson, and classroom } \\
\text { management), }\end{array}$} \\
\hline \multirow{2}{*}{$\begin{array}{l}\text { Methodisches Repertoire: } \\
\text { - Lehr-Lern-Methoden und-Konzepte und deren } \\
\text { lernzieladäquate Orchestrierung } \\
\text { - Generelle Prinzipien der Individual- und } \\
\text { Lernprozessdiagnostik und Evaluation }\end{array}$} & \\
\hline & $\begin{array}{l}\text { assessment } \\
\text { (including diagnosing principles irrespective of } \\
\text { the subject, evaluation procedures) }\end{array}$ \\
\hline
\end{tabular}

Abb. 1 Definitionen zentraler Inhaltsbereiche pädagogischen Wissens aus Überblicksbeiträgen

und Evaluation einen Unterpunkt ausmachen, der bei König (2014) mit assessment eigens ausgewiesen ist.

Trotz ihrer Übereinstimmungen bleibt bei beiden Überblicksarbeiten der Anschluss von Fachlichkeit theoretisch unterbestimmt. Während Aspekte der Klassenführung als relativ fächerübergreifende Herausforderung des Unterrichtens betrachtet (z. B. Praetorius et al. 2016) und somit auch ohne fachliche Konkretisierung gedacht werden können, dürfte beispielsweise für die adäquate Berücksichtigung kognitiver Lernvoraussetzungen als Teil von Unterrichtsstrategien zum Umgang mit Heterogenität nicht allein generisches, sondern auch domänenspezifisches Wissen nötig sein (z. B. Bremerich-Vos 2017).

\subsubsection{Fachdidaktisches Wissen}

Ausgehend von einer Kritik an einer vorwiegend fächerübergreifenden Betrachtung unterrichtlicher Herausforderungen und ausgehend von der Bemühung, Fachliches und Pädagogik in eine ausgewogene Balance zu bringen, betonte Shulman (1986) bereits Mitte der 1980er-Jahre die Notwendigkeit eines Fokus auf ,teachers' cognitive understanding of subject matter content and the relationships between such understanding and the instruction teachers provide for students“ (Shulman 1986, S. 25). In der internationalen Bildungsforschung wurde in der Folge die Fachlichkeit von Unterricht zunehmend beachtet. So betonen sowohl theoretisch-konzeptionelle Arbeiten (im Überblick zuletzt Depaepe et al. 2013) als auch empirische Untersuchungen (u. a. Hill et al. 2005; Baumert et al. 2010) die besondere Bedeutung des fachdidaktischen Wissens von Lehrkräften für die Unterrichtsqualität und die Steigerung von Fachleistungen ihrer Schülerinnen und Schüler (vgl. im Überblick Blömeke 2004; Lipowsky 2006). Ohne fachdidaktisches Wissen, das - sehr kurz gefasst - die Lehrperson auf der kognitiven Ebene dazu befähigt, die „Sache“ in den pädagogischen Kontext des Lehrens und Lernens zu stellen (vgl. u. a. Shulman 
1986, 1987; Kennedy 1990), ist Unterricht kaum vorstellbar. Lehrkräfte sind auf fachdidaktisches Wissen angewiesen, um ihrer pädagogischen Verantwortung den Lernenden gegenüber und der gesellschaftlichen Aufgabe von Schule gerecht zu werden (KMK 2012). Für die Lehrerbildung haben Reformdiskurse kenntlich gemacht, dass im Bereich der fachdidaktischen Ausbildung ein großes Potenzial zur Optimierung der Ausbildung zukünftiger Lehrpersonen liegen kann (u. a. Expertenkommission 2007).

Es stellt sich die Frage, was fachdidaktisches Wissen bzw. PCK von Lehrpersonen ist. Dazu liegt eine Fülle von Arbeiten der letzten drei Jahrzehnte vor. Übersichtsarbeiten haben daher versucht, Gemeinsamkeiten zu identifizieren. Zusammenfassend zeigen viele Konzeptionen von PCK zumindest zwei Facetten: Wissen über Lehrmethoden des Faches (einschließlich Wissen um fachliche Darstellung und Erklärungen) und Wissen über das Lernen und Verständnis von Schülerinnen und Schüler, einschließlich der Antizipation von Verständnisschwierigkeiten von Schülerinnen und Schülern im betreffenden Fach (u. a. Van Driel et al. 1998; Park und Oliver 2008; Bukova-Güzel 2010).

Dabei dürften zwischen PCK in den bislang vornehmlich untersuchten Disziplinen Mathematik und Naturwissenschaften auf der einen und in den sprachlichen Fächern auf der anderen Seite erhebliche Differenzen bestehen. Sie betreffen zum einen die Abgrenzung von PCK und CK. So ist z. B. in Deutsch und Englisch der Inhalt zugleich Medium der Lehre, sodass hier die Unterscheidung von PCK und CK zumindest partiell anders als üblich zu fassen sein dürfte (z. B. Freeman 2002; Burns et al. 2015). Zum anderen sind die Konturen der Sprachfächer im Vergleich mit Mathematik und den Naturwissenschaften recht diffus, worauf bereits Grossman und Shulman (1994) hingewiesen haben. So gibt es z. B., was den Umgang mit literarischen Texten angeht, nicht nur zahlreiche miteinander konkurrierende Literaturtheorien, sondern auch eine Fülle von damit einmal mehr, einmal weniger korrespondierenden Unterrichtsmethoden (vgl. Kammler 2000).

Normativ betrachtet besteht PCK hier nicht nur aus Wissen im Hinblick auf diese Vielfalt der Zugänge zu literarischen Texten, sondern auch aus der Fähigkeit, von Fall zu Fall eine begründete Auswahl zu treffen. Das Wissen, das dabei im Spiel ist, beschreiben Grossman und Shulman (1994 S. 24) verallgemeinernd wie folgt: „The knowledge and practical understanding teachers act upon daily in classrooms is unlikely to be composed of principles derived from research, not even the precepts of dedicated teacher educators. Rather, teachers' knowledge is composed largely of a repertoire of cases, of what happened in particular classes with specific kids." Fälle sind Ereignisse in der Zeit und der ihnen angemessene Darstellungsmodus ist das Erzählen. Für Erzählungen konstitutive Elemente sind die Handelnden, deren Ziele, Motive und Mittel in spezifischen, auch als konflikthaft erlebten Situationen, in denen Entscheidungen zu treffen sind. Grossman und Shulman beziehen sich in diesem Kontext auf Bruners Unterscheidung von narrativem und paradigmatischem Wissens- bzw. Denkmodus (Bruner 1986, 1991). Anders als der narrative, Plausibilität mithilfe von Geschichten erzeugende Modus zielt der paradigmatische auf das Erklären bzw. Verifizieren und Falsifizieren mit den Mitteln logischer und mathematisch-naturwissenschaftlicher Prozeduren. 
Auch andere Forscher betonen die Relevanz des Erzählens im Rahmen dessen, was sie als praktisches Lehrerwissen bestimmen (z. B. Clandinin 1992). Sie beziehen sich aber wie z. B. Schön (1983) mit der Figur reflektierter Praktiker, die nicht als Anwender von Ergebnissen empirischer Forschung zu verstehen seien, auf Lehrpersonen im Allgemeinen, nicht auf Lehrende bestimmter Fächer. Ob z. B. das Lehrerwissen in sprachlichen Fächern im Vergleich mit dem in Mathematik und den naturwissenschaftlichen Fächern in besonderer Weise narrativ strukturiert ist, ob letzteres in der Redeweise Bruners nicht angemessener als Amalgam narrativen und paradigmatischem Wissens zu verstehen ist, bleibt an dieser Stelle offen. Das hat unseres Erachtens damit zu tun, dass das Konstrukt des praktischen Lehrerwissens bislang noch zu vage ist, wie u. a. Fenstermacher (1994, S. 24) betont: „[There is a] lack of precision in the concept of practical knowledge, as it is used across the several teaching research programs."

PCK dürfte jedenfalls für die verschiedenen Domänen unterschiedlich zu definieren sein, was auf allgemeiner Ebene u. a. zu Schwierigkeiten bei der Konstruktdefinition sowie bei der Messbarkeit führen kann (u. a. Park und Oliver 2008; König 2014; König et al. 2016). Es ergibt sich u. a. die Frage, inwieweit fächerübergreifendes Wissen von Lehrkräften dann je nach Domäne unterschiedlich eng an das fachdidaktische Wissen anschließt.

\subsubsection{Zum Verhältnis von pädagogischem und fachdidaktischem Wissen}

Fachdidaktisches Wissen wird als eine Art „Amalgam“ von pädagogischem und fachlichem Wissen betrachtet (Shulman 1987, S. 8; vgl. auch Morine-Dershimer und Kent 1999). Allerdings ist es bislang eine weitgehend unbeantwortete Frage, inwieweit pädagogisches und fachdidaktisches Wissen voneinander konzeptuell und empirisch jeweils klar getrennt werden können. Einerseits lassen sich bestimmte didaktische Fragen konkret nur klären, wenn sie für eine bestimmte Domäne betrachtet werden, da unterrichtliche Lehr-Lernprozesse in der Schule ohne spezifische Inhalte nicht vorstellbar sind (u. a. Weinert 1994). Andererseits lassen sich bestimmte Unterrichtskonzepte auch auf einer allgemeinen Ebene betrachten wie z. B. die Klassenführung (vgl. z. B. Praetorius et al. 2016). Dieser Umstand sollte vor dem Hintergrund einer Lehramtsausbildung, in der die Zwei- oder Mehrfachausbildung die Regel ist, auch unter der Perspektive einer Ausbildungseffizienz betrachtet werden. So kann es hilfreich sein, allgemeine didaktische und lehr-lern-psychologische Prinzipien in der Ausbildung zu vermitteln, welche die angehenden Lehrpersonen für fachliche Situationen ihres Unterrichts konkretisieren. Benötigt wird hier allerdings die Unterstützung durch inhaltsspezifisch gestaltete Lerngelegenheiten. Diese werden strukturell von der jeweiligen Fachdidaktik verantwortet, nicht aber von fächerübergreifenden pädagogischen Fachbereichen übernommen (Blömeke 2002; Kunina-Habenicht et al. 2012). So erwerben Lehramtsstudierende professionelles Wissen in beiden Bereichen zunächst relativ getrennt; gefördert wird dies an den meisten Universitäten durch die auf unterschiedliche Fakultäten verteilten Verantwortlichkeiten, was in Reviews zum Reformbedarf als Problem der Fragmentierung der Lehrerbildung immer wieder kritisiert wurde (u. a. Merzyn 2002; Flores 2016). Mit dieser institutionellen Fragementierung dürfte auf Seiten der Studierenden eine 
kognitive „Fragmentierung“ korrespondieren. Bei berufstätigen Lehrkräften ist sie wahrscheinlich weniger deutlich oder gar nicht mehr auszumachen. Insbesondere die Forschung zur Lehrerexpertise betont ja die für Lehrkräfte mit hoher Expertise typische Vernetzung von Wissenselementen, während Novizen (z. B. Lehramtsstudierende) zunächst nur über eine gering strukturierte Wissensbasis verfügen (u.a. Bromme 1992; Hogan et al. 2003; Berliner 2004).

\subsection{Befunde aus bisheriger empirischer Forschung}

\subsubsection{Empirische Erfassung pädagogischen und fachdidaktischen Wissens}

Fachdidaktisches Wissen wurde zunächst für die Domäne der Mathematik bearbeitet (Ball et al. 2008; Baumert et al. 2010; Blömeke et al. 2010; Tatto et al. 2012), in jüngerer Zeit zudem für naturwissenschaftliche Fächer (u.a. Riese und Reinhold 2012; Großschedl et al. 2015; Lenske et al. 2015). Sprachliche Fächer wurden bisher nur sehr vereinzelt in den Blick genommen (für einen Überblick vgl. Gitomer und Ziske 2015), im deutschsprachigen Raum stellte das Projekt Teacher Education and Development Study - Learning to Teach (TEDS-LT; Blömeke et al. 2011; Bremerich-Vos und Dämmer 2013) einen ersten Meilenstein dar. Die dort begonnenen Arbeiten dienten als Basis für die Weiterentwicklung und Verbesserung fachdidaktischer Testungen in den Domänen Deutsch und Englisch. So wurde eine Testung englischdidaktischen Professionswissens im Projekt Professionelle Kompetenz von Englischlehrkräften (PKE; König et al. 2016) entwickelt, die anschlussfähig an den internationalen Forschungsstand ist. Die deutschdidaktische Testung wurde im Kontext der Arbeiten des Projekts Die Bedeutung des professionellen Wissens angehender Deutschlehrkräfte für ihre Planung von Unterricht (PlanvoLL-D; König et al. 2017) vorangebracht und in der vorliegenden Studie eingesetzt.

In den vergangenen Jahren gab es einen deutlichen Zuwachs an Forschung zur standardisierten Erfassung des pädagogischen Wissens (vgl. im Überblick König 2014; Voss et al. 2015). Der vorliegende Beitrag bezieht sich auf die Arbeiten, die im Kontext der internationalen Vergleichsstudie Teacher Education and Development Study - Mathematics (TEDS-M; Tatto et al. 2012) in den vergangenen Jahren entstanden sind, und baut auf diesen auf, insofern das im Rahmen von TEDS-M entwickelte Testinstrument zur Erfassung von pädagogischem Wissen auch in der vorliegenden Studie zum Einsatz gelangt. Weitere Informationen zur Operationalisierung von fachdidaktischem und pädagogischem Wissen erfolgen im Methodenteil (Abschn. 5).

\subsubsection{Analysen zur Beziehung von pädagogischem und fachdidaktischem Wissen}

Bisher wurde der Analyse der Beziehung zwischen pädagogischem Wissen auf der einen und fachdidaktischem Wissen in mehreren Disziplinen auf der anderen Seite nur wenig Beachtung geschenkt. Erste Analysen hierzu liegen aus dem Projekt Professionswissen von Lehrkräften in den Naturwissenschaften (Prowin; Lenske et al. 2015) für berufstätige Lehrkräfte mit den Fächern Biologie, Chemie und Physik vor. Lenske et al. (2015, S. 240) berichten über (manifeste) Korrelationen des pädago- 
gisch-psychologischen Wissens (operationalisiert über zwei Teilskalen: konditionalprozedurales und deklaratives Wissen) mit dem fachdidaktischen Wissen in Biologie, Chemie, Physik, die allesamt positiv sind, allerdings nach Fach und Art des pädagogisch-psychologischen Wissens zum Teil erheblich variieren. Am höchsten korreliert das konditional-prozedurale pädagogisch-psychologische Wissen mit dem fachdidaktischen Wissen bei Chemielehrkräften $(0,50)$, am niedrigsten mit dem fachdidaktischen Wissen von Physiklehrkräften $(0,19)$. Deklaratives pädagogisch-psychologisches Wissen ist sehr schwach mit chemiedidaktischem $(0,16, \mathrm{n}$. s.), dagegen signifikant mit physikdidaktischem Wissen korreliert $(0,31)$. Darüber hinaus ist das pädagogisch-psychologische Wissen mit dem fachdidaktischen Wissen systematisch höher als mit dem fachlichen Wissen korreliert.

Für angehende Lehrkräfte der zweiten Ausbildungsphase liegen korrelative Befunde für die Domänen Mathematik und Englisch vor. Im Rahmen der auf Referendarinnen und Referendare bezogenen Studie COACTIV-R, d. h. der Fortsetzungsstudie im Rahmen des Forschungsprogramms Professionswissen von Lehrkräften, kognitiv aktivierender Mathematikunterricht und die Entwicklung von mathematischer Kompetenz (COACTIV; Baumert und Kunter 2006; Kunter et al. 2011), wurde das pädagogisch-psychologische Wissen von angehenden Lehrkräften erfasst. Voss et al. (2011, S. 963) gelangen dabei zu einer höheren - latenten - Korrelation mit mathematikdidaktischem Wissen $(0,42)$ als mit mathematischem Fachwissen $(0,24)$. Dies entspricht in etwa der für TEDS-M berichteten Korrelation zwischen pädagogischem und mathematikdidaktischem Wissen (manifest: 0,30, unter Berücksichtigung einer Minderungskorrektur: 0,42). Die für Referendarinnen und Referendare mit Fach Englisch berichtete latente Korrelation zwischen pädagogischem und fachdidaktischem Wissen in PKE ist dagegen mit 0,77 viel höher (König et al. 2016, S. 328).

Insgesamt betrachtet ist somit der Stand der Forschung als wenig einheitlich zu bezeichnen, sodass weitere Zusammenhangsanalysen, vor allem auf latenter Ebene, angeraten erscheinen. Außerdem ist für angehende Lehrkräfte der ersten Ausbildungsphase die Frage nach dem Zusammenhang von pädagogischem und fachdidaktischem Wissen bislang kaum bearbeitet worden (vgl. König 2014; Voss et al. 2015). Eine Ausnahme stellt die Studie TEDS-LT dar, auf die die folgenden Analysen aufbauen (König et al. 2011), insofern weiterentwickelte Testinstrumente zum Einsatz gelangen.

\section{Fragestellungen und Hypothesen}

Im Mittelpunkt des vorliegenden Beitrags steht die Frage nach der Struktur des professionellen Wissens von Lehramtsstudierenden, und zwar am Beispiel von pädagogischem und fachdidaktischem Wissen der Kernfächer Deutsch, Englisch und Mathematik. Dies schließt auch eine Prüfung der Validität der verwendeten Wissenstests ein. Vor dem Hintergrund des dargestellten Forschungsstands fokussieren wir somit zwei Fragestellungen, die wir mit den folgenden Hypothesen (im Folgenden abgekürzt mit $\mathrm{H}$ ) bearbeiten: 


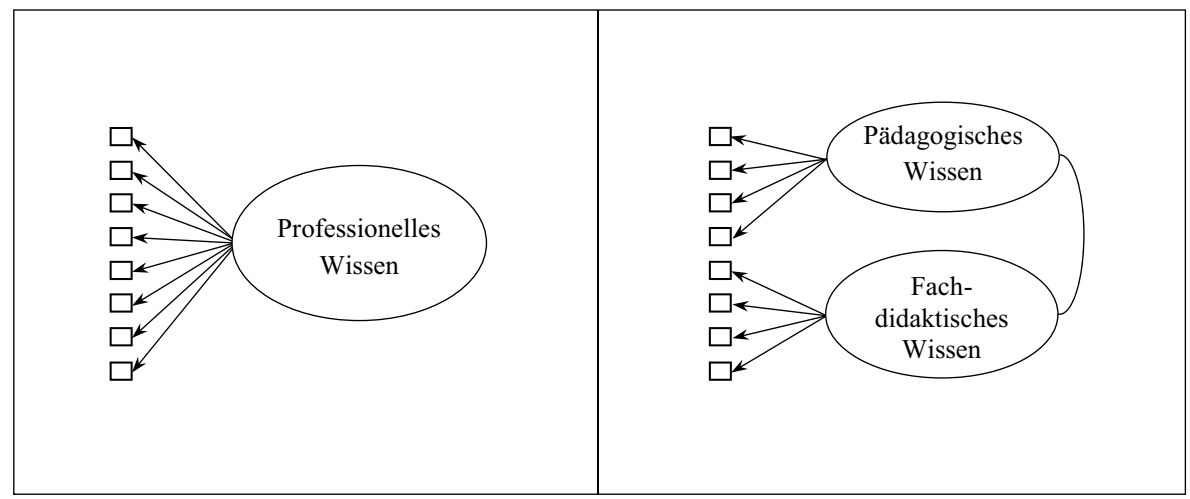

Abb. 2 Schematische Darstellung der Dimensionierung von pädagogischem und fachdidaktischem Wissen (Fragestellung 1)

1. Welche Struktur zeigt sich zwischen pädagogischem Wissen und fachdidaktischem Wissen von Lehramtsstudierenden in den Fächern Deutsch, Englisch und Mathematik?

Pädagogisches und fachdidaktisches Wissen sollten sich, so unsere erste Hypothese (H1a), für jede der drei hier in Rede stehenden Domänen empirisch trennen lassen. Denn einerseits handelt es sich bei der untersuchten Zielgruppe um Novizenlehrpersonen mit gering strukturierter Wissensbasis, andererseits werden Lerngelegenheiten in der Pädagogik und Fachdidaktik institutionell getrennt angeboten. Abb. 2 ist eine schematische Darstellung der Hypothesenprüfung zu entnehmen: Ein zweidimensionales Modell, das zwischen beiden Wissensbereichen unterscheidet (Abb. 2, rechte Hälfte), sollte demnach einem eindimensionalen Modell, das ,professionelles Wissen“ über einen Gesamtfaktor abbildet (Abb. 2, linke Hälfte), überlegen sein (gemessen mithilfe jeweiliger Modell-Fit-Indizes).

Aufgrund unterschiedlicher Fächerkulturen vermuten wir zudem, dass unterschiedlich hohe Interkorrelationen zwischen pädagogischem und fachdidaktischem Wissen in Abhängigkeit von der Domäne bestehen (H1b). Da möglicherweise pädagogisches Wissen ähnlich wie das Lehrerwissen in sprachlichen Fächern, aber anders als das Wissen in Mathematik und den naturwissenschaftlichen Fächern, in besonderer Weise narrativ strukturiert ist (vgl. unsere Ausführungen in Abschn. 2.2.2), vermuten wir höhere Korrelationen zwischen pädagogischem Wissen und dem englisch- bzw. deutschdidaktischen Wissen als zwischen pädagogischem Wissen und mathematikdidaktischem Wissen.

2. Zeigen sich Unterschiede im Niveau des erreichten Wissens bei Lehramtsstudierenden in Abhängigkeit von ihren Lerngelegenheiten?

Der Bearbeitung dieser Fragestellung legen wir ein Untersuchungsmodell zugrunde, das zwischen Lerngelegenheiten und Hintergrund- bzw. Eingangsmerkmalen der Lehramtsstudierenden unterscheidet (Abb. 3). Unser Fokus liegt auf der Analyse des Studienabschnittes sowie der bereits studierten Ausbildungsinhalte, während die 


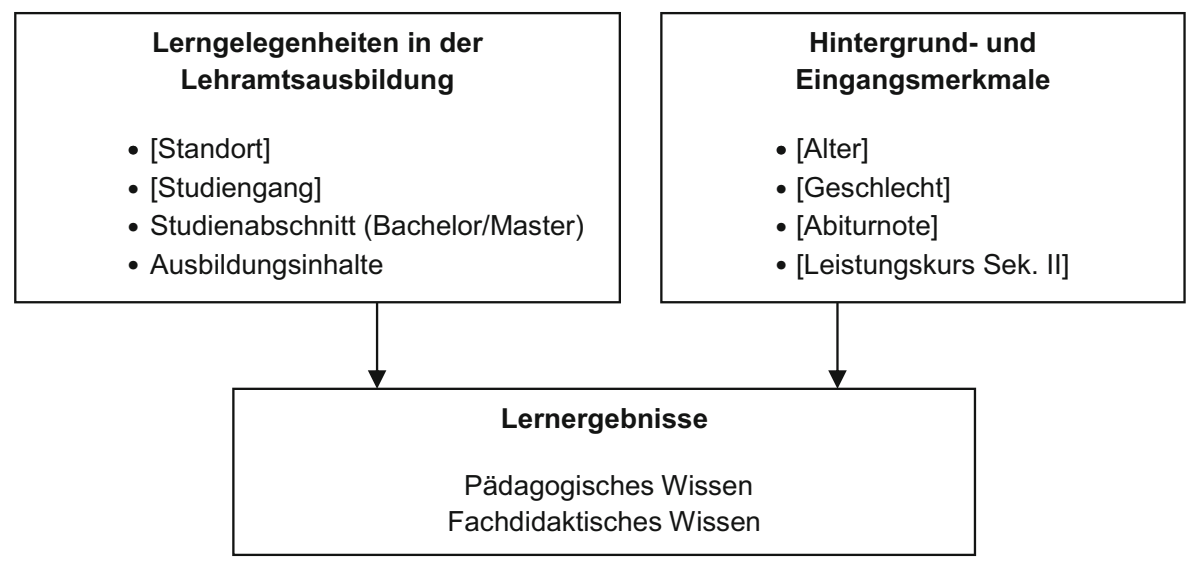

Abb. 3 Schematische Darstellung der Erklärung pädagogischen und fachdidaktischen Wissens (Fragestellung 2, Kontrollvariablen in eckigen Klammern)

übrigen Variablen (Standort, Lehramt, Hintergrund- und Eingangsmerkmale) lediglich als Kontrollvariablen einbezogen werden. ${ }^{5}$ Auf der institutionellen Seite beziehen wir den Standort sowie den Studiengang als Kontrollvariable ein, da bisherige Studien Variation im fachdidaktischen bzw. pädagogischen Wissen berichten, die auf diese beiden Merkmale der Lehramtsausbildung zurückgeführt werden konnten (Standort: z. B. Blömeke 2013; Studiengang: z. B. Kleickmann et al. 2013, für das fachdidaktische Wissen; sowie z. B. König und Klemenz 2015, für das pädagogische Wissen). Als Hintergrund- bzw. Eingangsmerkmale beziehen wir das Alter, das Geschlecht, die Abiturnote sowie den Besuch des Leistungskurses in der Sekundarstufe II ein. Vor allem für das Geschlecht (zugunsten weiblicher Studierende) und für die Abiturnote liegen aus bisherigen Studien (z. B. König und Seifert 2012; Blömeke 2013) relativ einheitliche Befunde zu ihrem jeweiligen Einfluss vor, sodass eine Kontrolle angebracht erscheint. Für das Alter sowie den Besuch des Leistungskurses sind die Befunde weniger eindeutig, wir beziehen sie jedoch ebenfalls in die nachfolgenden Analysen ein.

Mit einer ersten Hypothese (H2a) vermuten wir, dass Unterschiede nach Studienabschnitt (jeweils 2. Semester in der Bachelor- bzw. Masterphase) in allen drei fachdidaktischen Tests sowie im pädagogischen Wissen bestehen. Diese Unterschiede bleiben auch bei Kontrolle des Lehramtsstudiengangs und des Standortes sowie weiterer Variablen (z. B. Geschlecht, Abiturnote) erhalten, da von einer hohen curricularen Validität der Tests ausgegangen und auch angenommen wird, dass die Ausbildung lernwirksam ist, was letztlich in einem relativ besseren Abschneiden der Master-Studierenden münden sollte.

Mit einer zweiten Hypothese (H2b) vermuten wir, dass in Regressionsanalysen, in denen Indikatoren für fachdidaktische Lerngelegenheiten (Ausbildungsinhalte, vgl. nachfolgenden Methodenteil) als Prädiktoren verwendet werden, diese das jewei-

\footnotetext{
${ }^{5}$ Daraus ergibt sich eine vereinfachte, schematische Darstellung für die vorliegende Studie, die z. B. von Wechselwirkungen zwischen Eingangsmerkmalen und Lerngelegenheiten abstrahiert.
} 
lige fachdidaktische Wissen vorhersagen (im Sinne einer konvergenten Validität), während sie pädagogisches Wissen nur schwach bzw. nicht vorhersagen können (im Sinne einer diskriminanten Validität).

\section{Methode}

\subsection{Stichprobe}

Zur Bearbeitung unserer Fragestellungen verwenden wir Daten von 889 Lehramtsstudierenden der beiden Volluniversitäten Hamburg und Köln, die im Sommer 2016 befragt bzw. getestet wurden. Es handelt sich um einen Teildatensatz der an beiden Universitäten im Rahmen ihrer jeweiligen Projekte der „Qualitätsoffensive Lehrerbildung“ durchgeführten Kompetenzmessungen. ${ }^{6}$ Dieser Teildatensatz besteht aus angehenden Lehrkräften, die sich für den Deutsch-, Englisch- und/oder Mathematikunterricht in der Sekundarstufe qualifizieren. Zum Zeitpunkt der Datenerhebung befanden sie sich entweder im zweiten Semester ihres Bachelor- oder im zweiten Semester ihres Master-Studiums. Es handelt sich somit um jene Kohorten, welche im Herbst 2015 ihren Studiengang begonnen haben. Die Projekte haben zum Ziel, beide Kohorten im jährlichen Turnus wiederholt zu befragen bzw. zu testen, sodass Vergleiche zwischen den Studienabschnitten vorgenommen, zukünftig aber auch längsschnittlich Entwicklungen analysiert werden können.

Der Teildatensatz enthält insgesamt fünf der durch die KMK (2009) definierten Lehramtstypen, wobei am einzelnen Standort nur vier vertreten sind, da in Köln (bzw. im Bundesland Nordrhein-Westfalen) das reine Sekundarstufen-I-Lehramt (KMK-Typ 3), in Hamburg hingegen das stufenübergreifende Sekundarstufenlehramt (KMK-Typ 2) angeboten wird (vgl. Tab. 1). ${ }^{7}$ Studierende wurden entweder in Lehrveranstaltungen oder über einen Online-Survey bzw. eine Kombination aus Befragung vor Ort und Fortsetzung als Online-Survey erreicht und mithilfe von Honoraren zur Teilnahme motiviert. Angestrebt war eine Vollerhebung der Zielgruppe, die Rücklaufquote variiert zwischen rund $30 \%$ und rund $50 \%$ für Bachelorund Master-Studierende des jeweiligen Standortes (Bachelor-Studierende: 27,2\% in Hamburg und 50,9\% in Köln; Master-Studierende: 41,4\% in Hamburg und 53,9\% in Köln). ${ }^{8}$

\footnotetext{
${ }^{6}$ Beide Projekte, das Projekt Professionelles Lehrerhandeln zur Förderung fachlichen Lernens unter sich verändernden gesellschaftlichen Bedingungen (ProfaLe) der Universität Hamburg und das Projekt $\mathrm{Zu}$ kunftsstrategie Lehrer*innenbildung Köln - Heterogenität und Inklusion gestalten (ZuS) der Universität zu Köln, werden im Rahmen der gemeinsamen ,Qualitätsoffensive Lehrerbildung“ von Bund und Ländern aus Mitteln des Bundesministeriums für Bildung und Forschung gefördert.

7 Studierende des Grundschullehramts (KMK-Typ 1) wurden zwar in Köln ebenfalls in die Erhebungen einbezogen. Da die Analysen des vorliegenden Beitrags sich jedoch auf die fachliche Differenzierung in der Sekundarstufe beziehen, bleibt dieser Lehramtstyp im Folgenden unberücksichtigt.

8 Die beiden Standortstichproben unterscheiden sich im Verhältnis von Bachelor- und Master-Studierenden, was teilweise erhebungstechnische, teilweise populationsbedingte Gründe hat. Aus diesem Grund verzichten wir nachfolgend auf jegliche Form von Standortvergleichen und verwenden die Standortzugehörigkeit als Kontrollvariable.
} 
Tab. 1 Untersuchungsstichprobe ( $\mathrm{n}=889$ Lehramtsstudierende), gegliedert nach Standort, Lehramtstypen der KMK (2009) und Studienabschnitt (Bachelor/Master)

\begin{tabular}{|c|c|c|c|c|c|c|c|}
\hline \multirow[t]{2}{*}{$\begin{array}{l}\text { KMK- } \\
\text { Typ }\end{array}$} & \multirow[t]{2}{*}{ Bezeichnung } & \multicolumn{2}{|c|}{$\begin{array}{l}\text { Universität } \\
\text { Hamburg }\end{array}$} & \multicolumn{2}{|c|}{$\begin{array}{l}\text { Universität } \\
\text { zu Köln }\end{array}$} & \multicolumn{2}{|l|}{ Gesamt } \\
\hline & & $\begin{array}{l}\text { Studie- } \\
\text { rende } \\
\text { im Ba- } \\
\text { chelor }\end{array}$ & $\begin{array}{l}\text { Studie- } \\
\text { rende } \\
\text { im } \\
\text { Master }\end{array}$ & $\begin{array}{l}\text { Studie- } \\
\text { rende } \\
\text { im Ba- } \\
\text { chelor }\end{array}$ & $\begin{array}{l}\text { Studie- } \\
\text { rende } \\
\text { im } \\
\text { Master }\end{array}$ & $\begin{array}{l}\text { Studie- } \\
\text { rende } \\
\text { im } \mathrm{Ba-} \\
\text { chelor }\end{array}$ & $\begin{array}{l}\text { Studie- } \\
\text { rende } \\
\text { im } \\
\text { Master }\end{array}$ \\
\hline 2 & $\begin{array}{l}\text { Übergreifende } \\
\text { Lehrämter der Pri- } \\
\text { marstufe und al- } \\
\text { ler oder einzelner } \\
\text { Schularten des Se- } \\
\text { kundarbereichs I }\end{array}$ & 48 & 75 & - & - & 48 & 75 \\
\hline 3 & $\begin{array}{l}\text { Lehrämter für al- } \\
\text { le oder einzelne } \\
\text { Schularten des Se- } \\
\text { kundarbereichs I }\end{array}$ & - & - & 76 & 29 & 76 & 29 \\
\hline 4 & $\begin{array}{l}\text { Lehrämter für den } \\
\text { Sekundarbereich II } \\
\text { (allgemeinbildende } \\
\text { Fächer) oder für das } \\
\text { Gymnasium }\end{array}$ & 36 & 69 & 146 & 79 & 182 & 148 \\
\hline 5 & $\begin{array}{l}\text { Lehrämter für die } \\
\text { Sekundarstufe II (be- } \\
\text { rufliche Fächer) oder } \\
\text { für die beruflichen } \\
\text { Schulen }\end{array}$ & 11 & 16 & 7 & 3 & 18 & 19 \\
\hline 6 & $\begin{array}{l}\text { Sonderpädagogische } \\
\text { Lehrämter }\end{array}$ & 11 & 14 & 220 & 49 & 231 & 63 \\
\hline- & Gesamt & 106 & 174 & 449 & 160 & 555 & 334 \\
\hline
\end{tabular}

Die in Tab. 1 dargestellte Stichprobe umfasst Lehramtsstudierende, deren Fächerkombination mindestens eines der drei Kernfächer Deutsch, Englisch oder Mathematik enthält. Für alle 889 Studierende liegen Informationen zu demographischen Merkmalen und Basismerkmalen ihrer Ausbildung vor (u. a. Lehramt, Fächerkombination), $805(90,6 \%)$ von ihnen nahmen an der Testung pädagogischen Wissens teil (Hamburg: 89,3\%; Köln: 91,1\%). Testwerte für fachdidaktisches Wissen liegen jeweils für die Hälfte der Lehramtsstudierenden in unserer Stichprobe vor, die das betreffende Fach auch studieren. ${ }^{9}$ Diese relativ niedrige Ausschöpfungsquote ist bedingt durch das verwendete Design, in dessen Rahmen jeder Teilnehmerin bzw. jedem Teilnehmer eine fachdidaktische Testung in der Regel nur in einem ihrer bzw. seiner Studienfächer abverlangt wurde, um den Teilnahmeaufwand zu begren-

\footnotetext{
9 Dies sind 266 für das Fach Deutsch (von insgesamt 521 Studierenden mit Fach Deutsch, 51,2\%), 133 für das Fach Englisch (von insgesamt 271 mit Fach Englisch, 49,1\%) und 135 für das Fach Mathematik (von insgesamt 208 mit Fach Mathematik, 64,9\%).
} 
zen. Mit insgesamt 534 bearbeiteten fachdidaktischen Tests von 486 Personen $^{10}$ ist der Rücklauf $(54,7 \%)$ jedoch akzeptabel. ${ }^{11}$

Angesichts des Stichprobenausfalls bei den Tests zur Erfassung fachdidaktischen Wissens wurde eine Drop-out-Analyse durchgeführt, in der auf der Basis der Stichprobe mit gültigen Werten in den personenbezogenen Merkmalen und dem pädagogischen Wissenstest die Teilnahme an der fortgesetzten Erhebung online (kodiert als 1, Nichtteilnahme kodiert als 0) vorhergesagt wurde. Als Prädiktoren wurden einbezogen: Alter, Geschlecht, Abiturnote, Score im pädagogischen Wissen. Für die Hamburger Stichprobe ist keiner der Prädiktoren statistisch signifikant, für die Kölner Stichprobe ist lediglich das pädagogische Wissen mit $\beta=0,18$ statistisch signifikant $(p<0,001)$. Demnach haben in Köln tendenziell jene Lehramtsstudierenden an einer fachdidaktischen Testung teilgenommen, die bereits im pädagogischen Wissenstest etwas besser abgeschnitten haben. Der Drop-out ist aber nicht systematisch bedingt durch Alter, Geschlecht oder Abiturnote der Studierenden.

\subsection{Instrumente}

\subsubsection{Testung des pädagogischen Wissens}

Zur Erfassung des pädagogischen Wissens wurde der im Rahmen von TEDS-M entwickelte Test eingesetzt, und zwar als Kurzfassung für die universitäre Ausbildungsphase (König und Blömeke 2010). Mit dem Test werden unterrichtliche Herausforderungen, wie sie in der Forschung zur Unterrichtsqualität beschrieben

\begin{tabular}{|l|l|}
\hline $\begin{array}{l}\text { Fünf berufliche Anforderungen von } \\
\text { Lehrpersonen beim Unterrichten }\end{array}$ & Themen für die Operationalisierung \\
\hline Strukturierung von Unterricht & $\begin{array}{l}\text { - Komponenten- und prozessbezogene Planung } \\
\text { - Analyse von Unterricht } \\
\cdot \text { Curriculare Strukturierung von Unterricht }\end{array}$ \\
\hline Motivierung & $\begin{array}{l}\text { - Leistungsmotivation } \\
\text { - Motivierungsstrategien im Unterricht }\end{array}$ \\
\hline Umgang mit Heterogenität & $\begin{array}{l}\text { - Differenzierungsmaßnahmen } \\
\text { - Methodenvielfalt }\end{array}$ \\
\hline Klassenführung & $\begin{array}{l}\text { - Störungspräventive Unterrichtsführung } \\
\text { - effektive Nutzung der Unterrichtszeit }\end{array}$ \\
\hline Leistungsbeurteilung & $\begin{array}{l}\text { - Funktionen und Formen } \\
\text { - zentrale Kriterien } \\
\text { - Urteilsfehler }\end{array}$ \\
\hline
\end{tabular}

Abb. 4 Inhalte des Tests zum pädagogischen Wissen (König und Blömeke 2009, S. 504)

10 Hier gilt es zu beachten, dass an der Universität Hamburg 48 Studierende an zwei verschiedenen Fachdidaktiktests teilnahmen.

11 Hier ist zu beachten, dass an der Universität zu Köln 40 Studierende, für die in der vorliegenden Analyse kein Ergebnis aus fachdidaktischen Tests vorliegt, aufgrund ihrer Fächerkombination anstelle einer deutsch-, englisch- oder mathematikdidaktischen Testung an einer Testung in einer der folgenden Fachdidaktiken teilnahmen: Physik, Chemie, Biologie oder Französisch. Die Testung einer Person in zwei verschiedenen Fächern wurde nicht verlangt, um die Belastung der Lehramtsstudierenden bei der Testung in einem vertretbaren Umfang zu halten. 


\begin{tabular}{|c|c|c|}
\hline Dimension & Item-Beispiel & $\begin{array}{l}\text { Lösung / Mögliche } \\
\text { korrekte } \\
\text { Lösungsantwort }\end{array}$ \\
\hline \multirow[t]{2}{*}{ Motivation } & $\begin{array}{l}\text { Bei welchen der folgenden Fälle handelt es sich um eine intrinsische } \\
\text { Motivation, bei welchen um eine extrinsische Motivation? } \\
\text { Kreuzen Sie ein Kästchen pro Zeile an. } \\
\text { Intrinsische Motivation / Extrinsische Motivation }\end{array}$ & \\
\hline & 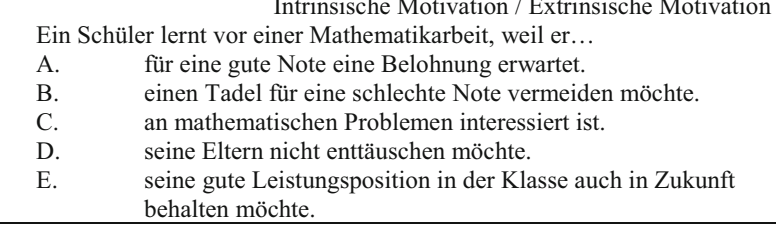 & $\begin{array}{l}\text { Intrinsische } \\
\text { Motivation: C; } \\
\text { Extrinsische } \\
\text { Motivation: A, B, } \\
\text { D, E }\end{array}$ \\
\hline $\begin{array}{l}\text { Umgang mit } \\
\text { Hetero- } \\
\text { genität }\end{array}$ & $\begin{array}{l}\text { Nennen Sie drei Unterrichtskonzepte bzw. Unterrichtsmethoden, die es den } \\
\text { Schüler(inne)n grundsätzlich ermöglichen, über die Differenzierung im } \\
\text { Unterricht mit zu entscheiden. } \\
\text { 1) } \\
\text { 2) } \\
\text { 3) }\end{array}$ & $\begin{array}{l}\text { 1) Wochenplan } \\
\text { 2) Stationenlernen } \\
\text { 3) Lernbuffet }\end{array}$ \\
\hline
\end{tabular}

Abb. 5 Beispiel-Testaufgaben zur Erfassung von pädagogischem Wissen (Quelle: König und Blömeke 2010)

werden, inhaltlich differenziert in die Inhaltsdimensionen Umgang mit Heterogenität, Strukturierung von Unterricht, Klassenführung, Motivierung und Leistungsbeurteilung. Jede der Dimensionen wird über mehrere komplexe Testaufgaben operationalisiert, die entsprechende unterrichtliche Herausforderungen zum Gegenstand haben (Abb. 4). Abb. 5 können außerdem Beispiel-Testaufgaben entnommen werden. Insgesamt wurden in der vorliegenden Analyse 13 Testaufgaben verwendet, aus denen 42 Testitems resultieren, die in die Skalierung eingeflossen sind.

\subsubsection{Testung des fachdidaktischen Wissens in den Fächern Deutsch, Englisch, Mathematik}

Zur Erfassung des deutschdidaktischen Wissens wurde ein Testinstrument eingesetzt, das zunächst im Rahmen des Projekts TEDS-LT (Blömeke et al. 2011) verwendet (Bremerich-Vos und Dämmer 2013) und dann im Projekt PlanvoLL-D (König et al. 2017) weiterentwickelt wurde. Es werden fünf Inhaltsbereiche unterschieden, die sich aus den Setzungen in den länderübergreifenden Bildungsstandards und den Kerncurricula für das Fach Deutsch ergeben: Sprechen und Zuhören, Schreiben, Rechtschreiben, Lesen und Sprache und Sprachgebrauch reflektieren. Im Bereich „Sprechen und Zuhören“ wird z. B. Wissen zu zentralen Differenzen von Mündlichkeit und Schriftlichkeit erfragt, in der Domäne Schreiben geht es u. a. um die Erhebung der Kenntnis von Methoden der Schreibförderung und von zentralen Merkmalen schulisch relevanter Textsorten bzw. -muster. Aufgaben zur Rechtschreibung zielen vor allem auf förderdiagnostisches Wissen, d. h. auf qualitative Analysen von Rechtschreibfehlern und darauf abgestimmte Förderstrategien. Aufgaben zum Kompetenzbereich Lesen haben mit Aspekten der als basal anzusehenden Leseflüssigkeit und mit Verfahren ihrer Förderung zu tun, darüber hinaus mit Lesestrategien und deren kontextsensibler Anwendung. In Lehrwerken gängige Aufgaben zu literarischen Texten sollen auf Modelle literarischen Lernens bezogen werden und es wird nach 
Wissen im Hinblick auf die Eignung und Anwendung von Methoden des handlungsund produktionsorientierten Umgangs mit literarischen Texten gefragt. Darüber hinaus sollen Lernaufgaben zu literarischen Texten als mehr oder weniger schwierig eingestuft werden. Die Testaufgaben zur Sprachreflexion beziehen sich vor allem auf den klassischen Kern der Schulgrammatik, d.h. auf die Lehre von Wortarten und Satzgliedern und auf Proben bzw. Operationen zu ihrer Bestimmung. Insgesamt handelt es sich um 29 Testaufgaben, die sich auf die fünf Dimensionen verteilen (zwischen drei und elf Testfragen pro Dimension). Aus jeder Testaufgabe resultiert ein Testitem für die Skalierung.

Fachdidaktisches Wissen für Englisch als Fremdsprache wurde mit einem Instrument erfasst, das im Projekt PKE (König et al. 2016) entwickelt wurde, ebenfalls auf Basis von Vorarbeiten aus TEDS-LT (Jansing et al. 2013). Der Test sieht drei Inhaltsdimensionen (curriculares Wissen, Wissen über Lehrstrategien, Wissen über das Schülerinnen-/Schülerwissen) vor, orientiert sich am internationalen Forschungsstand zum fachdidaktischen Wissen (u. a. Shulman 1987; Bukova-Güzel 2010) und folgt wichtigen Überlegungen zum fremdsprachendidaktischen Wissen (u. a. Gatbonton 1999; Borg 2006)..$^{12}$ Die Inhaltsdimension „curriculares Wissen“ umfasst bspw. Vorstellungen über den Kanon des Fachs Englisch abhängig von Schulform und Schulstufe; so sollten der thematische Aufbau des Curriculums und Definitionen von Kompetenzen bekannt und hierauf aufbauend die Fähigkeit der Analyse und begründeten Auswahl von Unterrichtsmaterialien vorhanden sein. Das „Wissen über Lehrstrategien" beinhaltet bspw. das Generieren von Handlungsoptionen für Erklärungen und Instruktionen im Englischunterricht und die Anwendung von Unterrichtsmethoden auf Problemstellungen des Lehrens im Fremdsprachenunterricht. Das „Wissen über Schülerinnen-/Schülerwissen“ bezieht sich auf die Kenntnis von Prozessen des Verstehens und (Fehl-)Vorstellungen der Schülerinnen und Schüler beim Spracherwerb und die Entwicklung von Handlungsoptionen bei der Fehlerkorrektur. Der Test umfasst 33 Testaufgaben, die sich relativ gleichmäßig auf die drei Inhaltsdimensionen verteilen (elf, zehn bzw. zwölf Items). Analog zum deutschdidaktischen Test resultiert aus jeder Testaufgabe ein Testitem für die Skalierung.

Mathematikdidaktisches Wissen wurde mithilfe des im Kontext von TEDS-M entstandenen Instruments TEDS-shortM erfasst (Buchholtz et al. 2016). Es umfasst zwei Inhaltsdimensionen: Stoffdidaktik und Unterrichtsdidaktik und im Anschluss an Anderson und Krathwohl (2001) eine Ausdifferenzierung der zugrundeliegenden kognitiven Prozesse in drei Wissensdimensionen: Erinnern und Abrufen, Verstehen und Anwenden, Bewerten und Generieren von Handlungsoptionen. Die stoffdidaktische Dimension umfasst u. a. stofflich geprägte Fragestellungen des Lehrens und Lernens von Mathematik und eine fachlich geprägte Diagnostik von Schülerlösungen. Die unterrichtsdidaktische Dimension umfasst Konzepte mathematischer Bildung, Leistungsbewertung im Mathematikunterricht sowie eine psychologisch

\footnotetext{
12 Im Unterschied zum deutschdidaktischen Test wurde also weniger von den fachlichen Inhaltsbereichen, sondern stärker von unterrichtsbezogenen Anforderungen ausgegangen. Gleichwohl berücksichtigt der Test zur Erfassung fachdidaktischen Wissens für Englisch als Fremdsprache die vier fachlichen Inhaltsbereiche Lesen, Schreiben, Sprechen, Zuhören (die sogenannten four skills; z. B. Hinkel 2006), sodass im Sinne der Gemeinsamkeit von sprachlichen Domänen eine gewisse Vergleichbarkeit beider fachdidaktischer Tests gegeben sein dürfte.
} 


\begin{tabular}{|c|c|c|}
\hline Dimension & Item-Beispiel & $\begin{array}{l}\text { Lösung/mögliche } \\
\text { korrekte Antwort }\end{array}$ \\
\hline Rechtschreiben & $\begin{array}{l}\text { Sie übernehmen eine 7. Klasse und wollen sich schnell über die } \\
\text { Rechtschreibfähigkeiten der Schüler*innen informieren. Sie schreiben } \\
\text { einen kleinen Test, in dem einzelne Wörter in Lücken und einige ganze } \\
\text { Sätze zu schreiben sind. Es kommen u.a. die folgenden Wörter vor: } \\
\text { Verkehrsschild, Tennisschläger, Bankräuber, Spinnennetz, } \\
\text { Schiedsrichter. Thomas schreibt sie so: Verkehrsschilt, Tennischläger, } \\
\text { Bankreuber, Spinnenetz, Schietsrichter. Sie wollen Thomas gezielt } \\
\text { fördern, damit er solche Fehler nicht mehr macht. Was sollte Thomas in } \\
\text { erster Linie lernen? } \\
\text { A. Er sollte mehr auf die Lautung der Wörter achten. } \\
\text { B. Er sollte lernen, die Wörter in Silben zu zerlegen. } \\
\text { C. Er sollte sich jeweils die ganzen Wörter einprägen. } \\
\text { D. Er sollte lernen, die Wörter in Morpheme zu zerlegen. }\end{array}$ & $\begin{array}{l}\text { D. Wörter in } \\
\text { Morpheme } \\
\text { zerlegen }\end{array}$ \\
\hline $\begin{array}{l}\text { Sprache und } \\
\text { Sprachgebrauch } \\
\text { untersuchen }\end{array}$ & $\begin{array}{l}\text { Sie möchten Schüler*innen in einer 5. oder 6. Klasse erklären, warum } \\
\text { das Adjektiv schnell ein typischeres Adjektiv ist als prima. Wie sollte } \\
\text { Ihre Erklärung lauten? }\end{array}$ & $\begin{array}{l}\text { Schnell ist } \\
\text { flektierbar, } \\
\text { insbesondere } \\
\text { steigerbar, prima } \\
\text { nicht. }\end{array}$ \\
\hline Schreiben & $\begin{array}{l}\text { In Lehrwerken für den Deutschunterricht in der Sekundarstufe wird oft } \\
\text { zwischen einer linearen und einer dialektischen schriftlichen Erörterung } \\
\text { unterschieden. Bei welchen der folgenden Aufgaben wird eine lineare } \\
\text { und bei welchen eine dialektische Erörterung erwartet? } \\
\text { A. Warum sollten Jugendliche viel lesen? } \\
\text { B. Viele HauptschülerInnen bekommen keinen Ausbildungsplatz. Nenne } \\
\text { einige Ursachen. } \\
\text { C. Sollten Jugendliche einen eigenen Fernseher haben? } \\
\text { D. Zensuren für LehrerInnen! Setze Dich mit dieser Forderung } \\
\text { auseinander. }\end{array}$ & $\begin{array}{l}\text { Linear A. und } \\
\text { B.; dialektisch C. } \\
\text { und D. }\end{array}$ \\
\hline
\end{tabular}

Abb. 6 Beispiel-Testaufgaben zur Erfassung von deutschdidaktischem Wissen

geprägte Diagnostik von Fehlvorstellungen, Lehr- und Lernformen und Unterrichtsarrangements, Curricula und Bildungsstandards für den Mathematikunterricht. Die stoffdidaktische Dimension wird mit sieben Testaufgaben erhoben, wobei alle Items Multiple-Choice-Items sind. Nur eine Testaufgabe bezieht sich auf die Dimension Erinnern und Abrufen, jeweils drei Testaufgaben beziehen sich auf Verstehen und Anwenden bzw. Bewerten und Generieren von Handlungsoptionen. Die unterrichtsdidaktische Dimension umfasst acht Testaufgaben, davon zwei offene Aufgaben. Jeweils drei Testaufgaben beziehen sich auf Erinnern und Abrufen bzw. Bewerten und Generieren von Handlungsoptionen, zwei auf Verstehen und Anwenden. Damit geht es hier mehrheitlich um kognitiv anspruchsvollere Wissensdimensionen. Das Testinstrument fokussiert zur Absicherung der curricularen Validität auf zentrale mathematikdidaktische Gegenstandsbereiche, wie sie in den ,Standards für die Lehrerbildung im Fach Mathematik“ (DMV, GDM und MNU 2008) als fachdidaktische Anreicherung zu den Lehrerbildungsstandards der KMK (2008) beschrieben sind sowie auf das internationale Framework der TEDS-M-Studie (Tatto et al. 2012).

Den Abb. 6, 7 und 8 sind jeweils Beispiel-Testaufgaben zur Erfassung des fachdidaktischen Wissens in den drei Fächern zu entnehmen. Antworten auf offene Testfragen wurden durch geschulte Rater anhand der Auswertungsrichtlinien zum jeweiligen Test kodiert. Rund 20\% der Fragebögen wurden doppelt kodiert, um die Übereinstimmung unabhängig voneinander erteilter Urteile zu überprüfen. Die Inter-Rater-Reliabilitäten (Cohen's Kappa) liegen für alle Tests in einem sehr guten Bereich ( $\mathrm{M}_{\text {Kappa }}>$ 0,75, vgl. Wirtz und Caspar 2002). 


\begin{tabular}{|c|c|c|}
\hline Dimension & Item-Beispiel & $\begin{array}{l}\text { Lösung / Mögliche } \\
\text { Korrekte } \\
\text { Lösungsantwort }\end{array}$ \\
\hline $\begin{array}{l}\text { Curriculares } \\
\text { Wissen }\end{array}$ & $\begin{array}{l}\text { Provide an example of a lexical item which shows that the term } \\
\text { "word" is problematic when talking about vocabulary learning. } \\
\text { Provide an example. }\end{array}$ & kitchen floor \\
\hline $\begin{array}{l}\text { Wissen über } \\
\text { Lehr- } \\
\text { strategien }\end{array}$ & $\begin{array}{l}\text { You as an English teacher get your students involved in collecting } \\
\text { information on tattoos today and in the past - with a special focus on } \\
\text { the role of tattoos among the Maoris in New Zealand. The outcome } \\
\text { of this collection will be a presentation that will be displayed in your } \\
\text { school. Which is the best label for this way of teaching? } \\
\text { Tick the right box. } \\
\text { A. Suggestopedia } \\
\text { B. Communicative approach } \\
\text { C. Direct method } \\
\text { D. Task-based language learning }\end{array}$ & $\begin{array}{l}\text { D. Task-based } \\
\text { language learning }\end{array}$ \\
\hline $\begin{array}{l}\text { Wissen über } \\
\text { das Schüler-/ } \\
\text { Schülerinnen- } \\
\text { wissen }\end{array}$ & $\begin{array}{l}\text { Your students talk about their hobbies. One of them says, "My } \\
\text { favorite hobbies is football and music". } \\
\text { You do not want to explicitly correct the student. However, you also } \\
\text { want to give him feedback on his incorrect use of the form ("to be") } \\
\text { since other students might not notice that particular mistake and } \\
\text { simply copy it. } \\
\text { Provide an answer in direct speech addressed to the student. }\end{array}$ & $\begin{array}{l}\text { "Your favorite hobbies } \\
\text { are football and music, } \\
\text { aren't they?" }\end{array}$ \\
\hline
\end{tabular}

Abb. 7 Beispiel-Testaufgaben zur Erfassung von englischdidaktischem Wissen (Quelle: König et al. 2016)

\begin{tabular}{|c|c|c|}
\hline Dimension & Item-Beispiel & $\begin{array}{l}\text { Lösung / Mögliche } \\
\text { Korrekte } \\
\text { Lösungsantwort }\end{array}$ \\
\hline $\begin{array}{l}\text { Stoffdidaktisches } \\
\text { Wissen }\end{array}$ & $\begin{array}{l}\text { Sie planen einen realitätsbezogenen Unterrichtseinstieg zum } \\
\text { Thema Exponentialfunktionen. Welches der folgenden } \\
\text { Themen eignet sich für einen Einstieg in das Thema? } \\
\text { Kreuzen Sie ein Kästchen eignet sich/eignet sich nicht pro } \\
\text { Zeile an. } \\
\text { A. Handytarife } \\
\text { B. Radioaktiver Zerfall }\end{array}$ & $\begin{array}{l}\text { A. Eignet sich nicht } \\
\text { B. Eignet sich }\end{array}$ \\
\hline $\begin{array}{l}\text { Unterrichtsdidaktisches } \\
\text { Wissen }\end{array}$ & $\begin{array}{l}\text { Stellen Sie sich vor, Sie planen eine Stunde für eine Klasse } \\
\text { des achten Jahrgangs, in der Ihre Schüler und Schülerinnen } \\
\text { den Satz des Pythagoras beweisen sollen. Da die Klasse } \\
\text { leistungsmäßig sehr heterogen ist, wollen Sie drei } \\
\text { Aufgabentypen anbieten, die jeweils einem } \\
\text { Repräsentationsmodus von Bruner entsprechen: enaktiv, } \\
\text { ikonisch, symbolisch. } \\
\text { Ordnen Sie die folgenden Beispiele den drei Aufgabentypen } \\
\text { zu. } \\
\text { Kreuzen Sie ein Kästchen enaktiv/ikonisch/symbolisch pro } \\
\text { Zeile an. } \\
\text { A. Berechnungsbeweis, der auf binomischen Formeln beruht. } \\
\text { B. Ergänzungsbeweis mit Puzzle } \\
\text { C. Einzeichnen der Einheitsquadrate }\end{array}$ & $\begin{array}{l}\text { A. Symbolisch } \\
\text { B. Enaktiv } \\
\text { C. Ikonisch }\end{array}$ \\
\hline
\end{tabular}

Abb. 8 Beispiel-Testaufgabe zur Erfassung von mathematikdidaktischem Wissen (Quelle: Buchholtz et al. 2016)

\subsubsection{Lerngelegenheiten}

In die vorliegende Analyse beziehen wir Indikatoren zur Erfassung fachdidaktischer Lerngelegenheiten ein (vgl. Hypothese H2b). Lerngelegenheiten stellen ein zentrales Konzept der empirischen Bildungsforschung dar (Clift und Brady 2005; Tatto et al. 2012) und gelten als wichtige Indikatoren bei der Überprüfung der validen Erfassung 
von Lernergebnissen (Schmidt et al. 2011; Kunina-Habenicht et al. 2013; Floden 2015). Modelle zur Wirksamkeit der Lehrerbildung gehen im Sinne des AngebotsNutzungs-Konzepts (Fend 2008; Helmke 2012) davon aus, dass das von angehenden Lehrkräften am Ende ihrer Ausbildung erworbene professionelle Wissen ein Resultat darstellt aus der Interaktion zwischen ihren Lernvoraussetzungen bei Eintritt in die Ausbildung und den Lerngelegenheiten, auf die sie während ihrer Ausbildung stoßen und die sie nutzen (Blömeke et al. 2010, 2011; König und Seifert 2012). In TEDS-M und TEDS-Folgestudien wie das Projekt Entwicklung von berufsspezifischer Motivation und pädagogischem Wissen in der Lehrerausbildung (König und Klemenz 2015) und das Projekt PKE (König et al. 2017), aber auch in anderen Studien (z. B. Schmidt et al. 2011; Watson et al. 2012; Kunina-Habenicht et al. 2013) werden Lerngelegenheiten unter anderem über Inhalte definiert, mit denen Lernende sich bis zu einem bestimmten Zeitpunkt auseinandergesetzt haben (oder nicht). Diese Herangehensweise steht in der Tradition der großen Schulleistungsstudien (McDonnell 1995). Für den Bereich der Lehrerbildung konnten diese Studien feststellen, dass der Umfang curricularer Inhalte ein valider Prädiktor für erworbenes professionelles Wissen darstellen kann, auch bei Kontrolle von Eingangsvoraussetzungen der angehenden Lehrkräfte.

Im Anschluss an die Testung fachdidaktischen Wissens wurden die Lehramtsstudierenden in unserer Studie daher gebeten, anhand einer Liste mit fachdidaktischen Ausbildungsinhalten anzugeben, ob der jeweilige Inhalt in ihrer Lehramtsausbildung bislang behandelt worden war (kodiert als 1) oder nicht (kodiert als 0). Tab. 2 enthält eine Übersicht über die jeweils berücksichtigten Bereiche der fachdidaktischen Ausbildungsinhalte und ihre Operationalisierung als Befragungsskalen. Die Skalen bilden das gesamte Spektrum von Ausbildungsinhalten der jeweiligen fachdidaktischen Lehre wie sie an Universitäten typischerweise stattfindet ab und wurden jeweils in aufwändigen Prozessen von uns mit der Unterstützung von Fachvertreterinnen und -vertretern entwickelt. ${ }^{13}$ Im Falle der Fachdidaktik Deutsch, für die der Wissenstest eng am Curriculum inhaltlich dimensioniert ist (vgl. vorherigen Abschn. 4.2.2), besteht somit eine relativ enge Entsprechung zwischen den Inhaltsdimensionen des Tests und der Dimensionierung der fachdidaktischen Ausbildungsinhalte. Für die Fachdidaktik Englisch und die Fachdidaktik Mathematik zeigen sich dagegen Unterschiede, da sich die Lerngelegenheiten am Curriculum der Lehrerausbildung orientieren, während die Tests stärker anforderungsbezogen konzipiert sind (vgl. vorherigen Abschn. 4.2.2). Diese Unterschiede in der Testkonzeption und Dimensionierung von Testinhalten werden bei der Interpretation und Diskussion unserer Ergebnisse berücksichtigt.

\footnotetext{
${ }^{13}$ So wurde beispielsweise für die Erfassung der englischdidaktischen Inhalte im Rahmen der Studie Professionelle Kompetenz von Englischlehrkräften (PKE; König et al. 2016) zunächst Ausbildungsinhalte in der Fachdidaktik Englisch mittels einer Dokumentenanalyse zusammengestellt. Lehrbücher, die in Lehrveranstaltungen der Fachdidaktik eingesetzt werden, wurden dabei als potentiell wichtige Grundlage zur Ermittlung von Ausbildungsinhalten angesehen. Durch die Analyse gängiger Lehrbücher der Fachdidaktik Englisch konnten somit die in Tab. 2 ausgewiesenen sieben Bereiche der fachdidaktischen Ausbildungsinhalte identifiziert und anschließend in einer Befragung von Expertinnen und Experten der Fachdidaktik Englisch bestätigt werden (Lammerding und König in Vorbereitung).
} 
Tab. 2 Beispiel-Items zur Messung von Dimensionen fachdidaktischer Ausbildungsinhalte bei angehenden Lehrkräften und Reliabilität der Skalen (Cronbachs Alpha)

\begin{tabular}{|c|c|c|c|c|}
\hline $\begin{array}{l}\text { Fach- } \\
\text { didaktik }\end{array}$ & Dimension & Beispiel-Item & Items & $\begin{array}{l}\text { Cronbachs } \\
\text { Alpha }\end{array}$ \\
\hline \multirow[t]{7}{*}{ Deutsch } & $\begin{array}{l}\text { Sprechen und Zuhö- } \\
\text { ren }\end{array}$ & $\begin{array}{l}\text { Unterrichtskommunikation (Kom- } \\
\text { munikation im Deutschunterricht) }\end{array}$ & 4 & 0,58 \\
\hline & Schreiben & Prozessorientierte Schreibdidaktik & 8 & 0,81 \\
\hline & Lesen & Förderung der Lesekompetenz & 5 & 0,86 \\
\hline & $\begin{array}{l}\text { Lesen literarischer } \\
\text { Texte }\end{array}$ & Aufgaben im Literaturunterricht & 7 & 0,79 \\
\hline & Sprachreflexion & Grammatikwerkstatt & 3 & 0,51 \\
\hline & Curriculare Aspekte & Bildungsstandards im Fach Deutsch & 4 & 0,75 \\
\hline & Gesamtskala & & 31 & 0,92 \\
\hline \multirow[t]{8}{*}{ Englisch } & Sprachdidaktik & Language Awareness & 24 & 0,88 \\
\hline & Literaturdidaktik & Storytelling & 8 & 0,76 \\
\hline & Methodik & Task-based language learning & 10 & 0,81 \\
\hline & Mediendidaktik & Medienkompetenz/Media literacy & 8 & 0,72 \\
\hline & Kulturdidaktik & $\begin{array}{l}\text { Interkulturelle kommunikative } \\
\text { Kompetenz }\end{array}$ & 13 & 0,89 \\
\hline & $\begin{array}{l}\text { Fachdidaktische } \\
\text { Forschung }\end{array}$ & $\begin{array}{l}\text { Subjektive Theorien von Fremd- } \\
\text { sprachenlehrenden, z. B. Überzeu- } \\
\text { gungsmuster }\end{array}$ & 11 & 0,82 \\
\hline & $\begin{array}{l}\text { Curriculum/ } \\
\text { Beurteilung }\end{array}$ & $\begin{array}{l}\text { Gemeinsamer Europäischer Refe- } \\
\text { renzrahmen für Sprachen }\end{array}$ & 12 & 0,81 \\
\hline & Gesamtskala & & 86 & 0,96 \\
\hline \multirow[t]{7}{*}{ Mathematik } & Grundlagen & Didaktik der Algebra & 20 & 0,88 \\
\hline & Heterogenität & $\begin{array}{l}\text { Sprachsensibler Mathematikunter- } \\
\text { richt }\end{array}$ & 8 & 0,77 \\
\hline & Technologie & Mathematiklernen mit Apps & 6 & 0,69 \\
\hline & $\begin{array}{l}\text { Methodik/ } \\
\text { Unterrichtspraxis }\end{array}$ & $\begin{array}{l}\text { Problemorientierung im Mathema- } \\
\text { tikunterricht }\end{array}$ & 8 & 0,86 \\
\hline & Curriculum & $\begin{array}{l}\text { Lehrpläne für den Mathematikun- } \\
\text { terricht }\end{array}$ & 8 & 0,77 \\
\hline & $\begin{array}{l}\text { Fachdidaktische } \\
\text { Forschung }\end{array}$ & $\begin{array}{l}\text { TEDS-Studien oder COACTIV- } \\
\text { Studie }\end{array}$ & 11 & 0,75 \\
\hline & Gesamtskala & & 61 & 0,96 \\
\hline
\end{tabular}

Wie Tab. 2 entnommen werden kann, können sämtliche Items zur Erfassung der fachdidaktischen Inhalte in der jeweiligen Domäne aufsummiert werden und als Gesamtskala berichtet werden. Die jeweiligen Inhaltsdimensionen lassen sich aber auch überwiegend als reliable Subskalen messen. Lediglich „Sprechen und Zuhören“ sowie „Sprachreflexion“ als Subskalen der Deutschdidaktik weisen mit Cronbachs Alpha von 0,58 bzw. 0,51 hohe Messfehler auf. ${ }^{14}$ Dies wird nachfolgend bei der Ergebnisinterpretation berücksichtigt.

14 Dass Cronbachs Alpha bei der Subskala „Sprechen und Zuhören“ niedrig ausfällt, dürfte darin begründet sein, dass zwei der vier Items, nämlich „Mündlichkeit und Schriftlichkeit“ und „Bildungssprache“, plausiblerweise auch der Subskala „Schreiben“ zugeordnet werden könnten. Neben „Grammatikwerkstatt““ 
Tab. 3 Deskriptive Statistiken der Skalen zur Erfassung fachdidaktischer Ausbildungsinhalte

\begin{tabular}{|c|c|c|c|c|c|c|c|}
\hline \multirow[t]{2}{*}{ Fachdidaktik } & \multirow[t]{2}{*}{ Subskala } & \multicolumn{3}{|c|}{$\begin{array}{l}\text { Studierende im Bachelor } \\
\text { (2. Semester) }\end{array}$} & \multicolumn{3}{|c|}{$\begin{array}{l}\text { Studierende im } \\
\text { Master (2. Semester) }\end{array}$} \\
\hline & & M & SE & SD & M & SE & SD \\
\hline \multirow[t]{6}{*}{ Deutsch } & Sprechen und Zuhören & 0,22 & 0,02 & 0,26 & 0,57 & 0,02 & 0,24 \\
\hline & Schreiben & 0,12 & 0,02 & 0,20 & 0,47 & 0,02 & 0,24 \\
\hline & Lesen & 0,08 & 0,02 & 0,19 & 0,50 & 0,04 & 0,38 \\
\hline & $\begin{array}{l}\text { Lesen literarischer } \\
\text { Texte }\end{array}$ & 0,20 & 0,02 & 0,27 & 0,48 & 0,03 & 0,28 \\
\hline & Sprachreflexion & 0,15 & 0,02 & 0,25 & 0,32 & 0,03 & 0,30 \\
\hline & Curriculare Aspekte & 0,05 & 0,01 & 0,16 & 0,41 & 0,03 & 0,35 \\
\hline \multirow[t]{7}{*}{ Englisch } & Sprachdidaktik & 0,26 & 0,02 & 0,21 & 0,57 & 0,02 & 0,17 \\
\hline & Literaturdidaktik & 0,15 & 0,03 & 0,22 & 0,42 & 0,04 & 0,27 \\
\hline & Kulturdidaktik & 0,18 & 0,03 & 0,22 & 0,48 & 0,04 & 0,29 \\
\hline & Mediendidaktik & 0,13 & 0,02 & 0,21 & 0,28 & 0,03 & 0,24 \\
\hline & Methodik & 0,10 & 0,02 & 0,21 & 0,44 & 0,04 & 0,28 \\
\hline & $\begin{array}{l}\text { Curriculum/ } \\
\text { Beurteilung }\end{array}$ & 0,07 & 0,02 & 0,16 & 0,33 & 0,03 & 0,20 \\
\hline & $\begin{array}{l}\text { Fachdidaktische For- } \\
\text { schung }\end{array}$ & 0,07 & 0,02 & 0,18 & 0,39 & 0,03 & 0,20 \\
\hline \multirow[t]{6}{*}{ Mathematik } & Grundlagen & 0,17 & 0,03 & 0,22 & 0,45 & 0,05 & 0,17 \\
\hline & Heterogenität & 0,13 & 0,02 & 0,18 & 0,41 & 0,04 & 0,27 \\
\hline & Technologie & 0,12 & 0,02 & 0,23 & 0,29 & 0,03 & 0,22 \\
\hline & $\begin{array}{l}\text { Methodik/ } \\
\text { Unterrichtspraxis }\end{array}$ & 0,19 & 0,03 & 0,28 & 0,58 & 0,05 & 0,31 \\
\hline & Curriculum & 0,14 & 0,02 & 0,21 & 0,45 & 0,04 & 0,23 \\
\hline & $\begin{array}{l}\text { Fachdidaktische For- } \\
\text { schung }\end{array}$ & 0,12 & 0,02 & 0,16 & 0,34 & 0,03 & 0,23 \\
\hline
\end{tabular}

Anmerkung: Kennwerte zur Deutschdidaktik beruhen auf Angaben von 239 Studierenden (Bachelor: 133, Master: 106); Kennwerte zur Englischdidaktik auf Angaben von 135 Studierenden (Bachelor: 80, Master: 55); Kennwerte zur Mathematikdidaktik auf Angaben von 134 Studierenden (Bachelor: 61, Master: 44)

Zur Prüfung der Skalen wurden Mittelwertunterschiede nach Studienabschnitt berechnet (Tab. 3). Die Ergebnisse verdeutlichen, dass es bei fast allen Skalen erwartungsgemäß große Differenzen (d. h. eine Standardabweichung oder mehr) zwischen den hier betrachteten Kohorten gibt (Bachelor vs. Master): Master-Studierende im zweiten Semester geben im Durchschnitt an, etwa jedes zweite Thema bereits studiert zu haben, während Bachelor-Studierende deutlich weniger Themen angeben. Dies spricht für die curriculare Validität der hier verwendeten Skalen zur Erfassung der Lerngelegenheiten.

gehören zur Subskala „Sprachreflexion“ die Items „Schulgrammatik“ und „Integrativer Sprachunterricht“. Es ist denkbar, dass die Testteilnehmerinnen und -teilnehmer das dritte Item nicht speziell auf den Kompetenzbereich „Sprachreflexion“, sondern auf den gesamten Deutschunterricht jenseits des Literaturunterrichts bezogen haben. 


\subsection{Skalierung der Wissenstests}

Die Wissenstests wurden zunächst mithilfe der Software Conquest (Wu et al. 1997) getrennt für jeden Test und entlang der jeweils vorhandenen Auswertungsrichtlinie (König und Blömeke 2010; Buchholtz et al. 2016; König et al. 2016) skaliert. In einem eindimensionalen Modell wurde dabei jedem Testitem aufgrund seiner empirischen Häufigkeit („Lösungsquote“) ein Itemparameter (,Schwierigkeitsparameter“) und jeder befragten Person entsprechend dem von ihr gezeigten Antwortverhalten ein Parameter auf der latenten Variable Theta („,Fähigkeitsparameter“) zugewiesen. Tab. 3 enthält zentrale Kennwerte aus der IRT-Skalierung für die vier verwendeten Tests. Insgesamt erweisen sich alle Tests als reliabel und die Varianz der latenten Variable ist hinreichend. Item-Diskriminationswerte liegen mit durchschnittlich $>0,4$ bei allen Tests in einem guten bis sehr guten Bereich, der Weighted Mean Square jedes Items fällt in einen akzeptablen Bereich, meist zwischen 0,8 und 1,20 (vgl. Bond und Fox 2007). Insgesamt streuen die gemessenen Fähigkeitswerte jedes einzelnen Wissenstests über mehrere Logits, was als hinreichende Varianz interpretiert werden kann (vgl. Bond und Fox 2007).

Für die nachfolgenden Analysen wurden ferner die Testitems, welche eine Inhaltsdimension eines Tests abbilden, zu einer Subskala aufsummiert und als Indikatorvariable zur Prüfung der faktoriellen Struktur im Strukturgleichungsansatz mithilfe der Software Mplus (Muthén und Muthén 2010 ) verwendet (Verfahren des sog. ,,item parceling“, vgl. Bandalos und Finney 2009). Dies ist im vorliegenden Fall insofern ratsam, als die Modelle für die einzelnen Fachdidaktiken jeweils nur auf einer kleineren Teilstichprobe basieren. Für den deutschdidaktischen Test ergaben sich somit fünf (Sprechen und Zuhören, Schreiben, Rechtschreiben, Lesen und Sprache, Sprachgebrauch reflektieren), für den englischdidaktischen drei (curriculares Wissen, Wissen über Lehrstrategien, Wissen über das Schülerinnen-/Schülerwissen) und für den mathematikdidaktischen zwei Indikatorvariablen (stoffdidaktisches Wissen, unterrichtsdidaktisches Wissen). Für den Test zur Erfassung von pädagogischem Wissen wurden vier Indikatorvariablen gebildet (Umgang mit Heterogenität, Strukturierung von Unterricht, Klassenführung/Motivierung, Leistungsbeurteilung). ${ }^{15}$

\section{Ergebnisse}

\subsection{Ergebnisse aus Strukturanalysen}

Zur Bearbeitung unserer ersten Fragestellung wurden für jede Domäne getrennt zwei Strukturmodelle berechnet (vgl. die schematische Darstellung in Abb. 2): ein eindimensionales Modell, das gesamthaft „,professionelles Wissen“ misst und nicht von

\footnotetext{
15 Die Reliabilitäten wurden zuvor mithilfe mehrdimensionaler Skalierungen (basierend auf den eindimensionalen Skalierungen, über die in Tab. 4 berichtet wird) mithilfe der Software Conquest geprüft. Sie liegen mit wenigen Ausnahmen im zufriedenstellenden bis guten Bereich für die einzelnen Subskalen der Tests (Deutschdidaktik: 0,59/0,75/0,74/0,76/0,73; Englischdidaktik: 0,80/0,79/0,83; Mathematikdidaktik: 0,71/0,73; Pädagogik: 0,84/0,83/0,67/0,59).
} 
Tab. 4 Statistische Kennwerte aus den Skalierungsanalysen der Tests

\begin{tabular}{llllll}
\hline Test & $\begin{array}{l}\text { Item- } \\
\text { Anzahl }\end{array}$ & $\begin{array}{l}\text { EAP-Re- } \\
\text { liabilität }\end{array}$ & $\begin{array}{l}\text { Theta- } \\
\text { Varianz }\end{array}$ & $\begin{array}{l}\text { Weighted } \\
\text { Mean Square } \\
\text { (min.-max.) }\end{array}$ & $\begin{array}{l}\text { Item-Dis- } \\
\text { crimination } \\
\text { (Mittelwert) }\end{array}$ \\
\hline $\begin{array}{l}\text { Pädagogisches Wissen } \\
\begin{array}{l}\text { Deutschdidaktisches } \\
\text { Wissen }\end{array}\end{array}$ & 42 & 0,88 & 1,06 & $0,73-1,24$ & 0,41 \\
$\begin{array}{l}\text { Englischdidaktisches } \\
\begin{array}{l}\text { Wissen } \\
\text { Mathematikdidaktisches }\end{array}\end{array}$ & 29 & 0,87 & 1,16 & $0,87-1,18$ & 0,42 \\
\begin{tabular}{l} 
Wissen \\
\hline
\end{tabular} & 27 & 0,88 & 1,03 & $0,80-1,23$ & 0,42 \\
\hline
\end{tabular}

einer Differenzierung in pädagogisches und fachdidaktisches Wissen ausgeht, sowie ein zweidimensionales Modell, das jeweils zwischen beiden Wissensbereichen unterscheidet. Insgesamt wurden somit sechs Modelle berechnet. Die aus den jeweiligen Spezifikationen resultierenden Modell-Fit-Indizes sind Tab. $5 \mathrm{zu}$ entnehmen. ${ }^{16} \mathrm{Da}$ die Modelle auf Basis der für jeden Test gebildeten Item-Parcels berechnet wurden, beinhalten die beiden deutschdidaktisch-pädagogischen Modelle neun, die beiden englischdidaktisch-pädagogischen sieben und die beiden mathematikdidaktisch-pädagogischen Modelle sechs Indikatorvariablen (vgl. vorherigen Abschn. 4.3).

Für jede Domäne lässt sich anhand der Indizes feststellen, dass eine empirische Trennung des professionellen Wissens in zwei Dimensionen einem eindimensionalen Modell überlegen ist. Pädagogisches und fachdidaktisches Wissen sind - trotz unterschiedlicher Domänen und Operationalisierungen - zwei Konstrukte. Entgegen unserer zweiten Hypothese (H1b) zeigen sich jedoch keine Unterschiede in der Höhe der latenten Korrelation zwischen pädagogischem und fachdidaktischem Wissen nach Domäne: Auch das mathematikdidaktische Wissen korreliert mit 0,64 ähnlich hoch wie das fachdidaktische Wissen in den Sprachen mit dem pädagogischen Wissen $(0,55$ bzw. 0,65$)$.

\subsection{Ergebnisse aus Pfadanalysen}

Zur Beantwortung unserer zweiten Fragestellung wurde für jede Domäne ein Pfadmodell spezifiziert (Tab. 6). Als abhängige Variablen dienten das pädagogische Wissen sowie das fachdidaktische Wissen in Deutsch (Modell 1), Englisch (Modell 2) und Mathematik (Modell 3). Die abhängigen Variablen wurden entsprechend der zweidimensionalen Modelle (Tab. 5) modelliert. Darauf aufbauend wurden entsprechend der Prüfung unserer Hypothesen H2a und H2b der Ausbildungsabschnitt sowie die spezifischen Ausbildungsinhalte der jeweiligen Fachdidaktik als Prädikto-

\footnotetext{
${ }^{16}$ Den Modell-Fit bewerten wir mithilfe verschiedener Indizes: anhand des Quotienten $\chi^{2} / \mathrm{df}$ (wobei Werte $<2$ einen sehr guten, $<3$ einen guten, $<4$ noch einen akzeptablen Modell-Fit indizieren); anhand des Comparative Fit Index (CFI, bei dem Werte $\geq 0,95$ einen sehr guten, $\geq 0,90$ einen guten, $<0,90$ einen schlechten Modell-Fit indizieren, vgl. Kline 2005); und anhand globaler Fit-Indizes (Root Mean Square Error of Approximation, RMSEA, sowie Standardized Root Mean Square Residual, SRMR), bei denen Werte $\leq 0,05$ einen sehr guten, Werte $\leq$ 0,08 noch einen guten Modell-Fit indizieren, vgl. Hu und Bentler (1999)
} 
Tab. 5 Modell-Fit für eindimensionale vs. zweidimensionale Modelle pädagogischen und fachdidaktischen Wissens

\begin{tabular}{|c|c|c|c|c|c|c|}
\hline & \multicolumn{2}{|c|}{$\begin{array}{l}\text { Pädagogisches und } \\
\text { deutschdidaktisches Wis- } \\
\text { sen }\end{array}$} & \multicolumn{2}{|c|}{$\begin{array}{l}\text { Pädagogisches und eng- } \\
\text { lischdidaktisches Wissen }\end{array}$} & \multicolumn{2}{|c|}{$\begin{array}{l}\text { Pädagogisches und mathe- } \\
\text { matikdidaktisches Wissen }\end{array}$} \\
\hline & $\begin{array}{l}\text { Ein-dim. } \\
\text { Modell }\end{array}$ & $\begin{array}{l}\text { Zwei-dim. } \\
\text { Modell }\end{array}$ & $\begin{array}{l}\text { Ein-dim. } \\
\text { Modell }\end{array}$ & $\begin{array}{l}\text { Zwei-dim. } \\
\text { Modell }\end{array}$ & $\begin{array}{l}\text { Ein-dim. } \\
\text { Modell }\end{array}$ & $\begin{array}{l}\text { Zwei-dim. } \\
\text { Modell }\end{array}$ \\
\hline $\begin{array}{l}\text { Korrelation } \\
\text { der Dimen- } \\
\text { sionen }\end{array}$ & - & $0,55^{* * *}$ & - & $0,65^{* * *}$ & - & $0,64 * * *$ \\
\hline \multicolumn{7}{|l|}{ Fit-Indizes } \\
\hline$\chi^{2}$ & $185,654 * * *$ & $49,309 *$ & $79,351 * * *$ & 14,047 & $31,921 * * *$ & 14,715 \\
\hline df & 27 & 26 & 14 & 13 & 9 & 8 \\
\hline$\chi^{2} / \mathrm{df}$ & 6,88 & 1,90 & 5,67 & 1,08 & 3,55 & 1,84 \\
\hline CFI & 0,831 & 0,975 & 0,857 & 0,998 & 0,902 & 0,971 \\
\hline RMSEA & 0,110 & 0,043 & 0,135 & 0,018 & 0,114 & 0,065 \\
\hline SRMR & 0,088 & 0,052 & 0,116 & 0,044 & 0,061 & 0,042 \\
\hline AIC & $12.197,089$ & $12.062,744$ & 5983,869 & 5920,565 & 4034,794 & 4019,588 \\
\hline
\end{tabular}

Anmerkung: ${ }^{*} p<0,05, * * * p<0,001$

CFI Comparative Fit Index, RMSEA Root Mean Square Error of Approximation, SRMR Standardized Root Mean Square Residual, AIC Akaike Information Criterion

ren einbezogen. Als Kontrollvariablen dienten auf institutioneller Seite der Standort sowie das Lehramt, auf individueller Seite wurden Hintergrund- und Eingangsmerkmale (Alter, Abiturnote, Geschlecht, Leistungskursbesuch; zur Kodierung vgl. die Anmerkung unterhalb Tab. 6) als zusätzliche Kontrollvariablen einbezogen. Die Ausbildungsinhalte wurden jeweils als Gesamtskala einbezogen (vgl. Tab. 2).

Auf Basis der Kennwerte in Tab. 6 lässt sich unsere erste Hypothese H2a für alle Domänen und sowohl für das pädagogische als auch das fachdidaktische Wissen bestätigen: Master-Studierende schneiden in allen Bereichen deutlich besser ab als Bachelor-Studierende. Die Pfadkoeffizienten für das pädagogische Wissen in den drei Modellen $(0,47 / 0,40 / 0,52)$ indizieren einen Effekt, der mindestens eine mittlere praktische Bedeutsamkeit besitzt. Für das fachdidaktische Wissen in Deutsch $(0,28)$ und Englisch $(0,30)$ sind die Effekte von (knapp) mittlerer praktischer Bedeutsamkeit, für das mathematikdidaktische Wissen $(0,51)$ liegt der Effekt sogar noch höher.

Unsere zweite Hypothese H2b lässt sich teilweise bestätigen. Spezifische fachdidaktische Ausbildungsinhalte in Deutsch, Englisch und Mathematik können - über den Ausbildungsabschnitt hinaus - einen Beitrag leisten zur Erklärung inter-individueller Unterschiede in den getesteten Leistungen im jeweiligen Fachdidaktiktest. Im ersten Pfadmodell beträgt der Koeffizient für das deutschdidaktische Wissen 0,15 (n. s.), im zweiten Pfadmodell für das englischdidaktische Wissen 0,32 ( $p<$ 0,01 ) und im dritten für das mathematikdidaktische Wissen 0,10 (n. s.). Die Pfadkoeffizienten sind also positiv, d. h. mit umfangreicheren Inhalten geht eine etwas bessere Testleistung einher. Sie sind aber nur im zweiten Modell statistisch signifikant und dort von mittlerer praktischer Bedeutsamkeit. Dabei fällt aber auf, dass die mathematikdidaktischen Inhalte $(0,24, p<0,05)$ auch das pädagogische Wissen 
Tab. 6 Kennwerte aus Pfadmodellen zur Erklärung von pädagogischem und fachdidaktischem Wissen

\begin{tabular}{|c|c|c|c|c|c|c|}
\hline \multirow[b]{2}{*}{$\begin{array}{l}\text { Abhängige } \\
\text { Variablen }\end{array}$} & \multicolumn{2}{|c|}{$\begin{array}{l}\text { Modell 1: } \\
\text { Pädagogisches und } \\
\text { deutschdidaktisches } \\
\text { Wissen }\end{array}$} & \multicolumn{2}{|c|}{$\begin{array}{l}\text { Modell 2: } \\
\text { Pädagogisches und } \\
\text { englischdidaktisches } \\
\text { Wissen }\end{array}$} & \multicolumn{2}{|c|}{$\begin{array}{l}\text { Modell 3: } \\
\text { Pädagogisches und mathe- } \\
\text { matikdidaktisches Wissen }\end{array}$} \\
\hline & $\begin{array}{l}\text { Pädago- } \\
\text { gisches } \\
\text { Wissen }\end{array}$ & $\begin{array}{l}\text { Deutsch- } \\
\text { didak- } \\
\text { tisches } \\
\text { Wissen }\end{array}$ & $\begin{array}{l}\text { Pädago- } \\
\text { gisches } \\
\text { Wissen }\end{array}$ & $\begin{array}{l}\text { Englisch- } \\
\text { didak- } \\
\text { tisches } \\
\text { Wissen }\end{array}$ & $\begin{array}{l}\text { Pädago- } \\
\text { gisches } \\
\text { Wissen }\end{array}$ & $\begin{array}{l}\text { Mathematik- } \\
\text { didaktisches } \\
\text { Wissen }\end{array}$ \\
\hline \multicolumn{7}{|l|}{ Pfadkoeffizienten } \\
\hline Standort & $0,20 * *$ & 0,06 & $0,44 * * *$ & 0,16 & 0,13 & $-0,09$ \\
\hline Lehramt & $-0,13^{*}$ & 0,10 & $-0,08$ & $-0,08$ & $-0,01$ & $-0,08$ \\
\hline Studienabschnitt & $0,47 * * *$ & $0,28 * *$ & $0,40 * * *$ & $0,30 * *$ & $0,52 * * *$ & $0,51 * * *$ \\
\hline Ausbildungsinhalte & 0,14 & 0,15 & 0,14 & $0,32 * *$ & $0,24 *$ & 0,10 \\
\hline Alter & $0,22 * *$ & 0,17 & 0,12 & $0,23 *$ & 0,14 & $0,21 *$ \\
\hline Geschlecht & $-0,02$ & $-0,10$ & $-0,14$ & $-0,19 *$ & 0,05 & 0,12 \\
\hline Abiturnote & $-0,13^{*}$ & $-0,35 * * *$ & $-0,19$ & $-0,35 * * *$ & $-0,15$ & $-0,30 * *$ \\
\hline $\begin{array}{l}\text { Leistungskurs } \\
\text { Sek. II }\end{array}$ & 0,02 & $-0,08$ & 0,01 & $-0,05$ & 0,00 & $-0,07$ \\
\hline $\mathrm{R}^{2}$ & 0,70 & 0,48 & 0,73 & 0,64 & 0,71 & 0,51 \\
\hline $\begin{array}{l}\text { Korrelation der } \\
\text { beiden abhängi- } \\
\text { gen Variablen }\end{array}$ & $0,29 *$ & & $0,41^{*}$ & & 0,23 & \\
\hline \multicolumn{7}{|l|}{ Fit-Indizes } \\
\hline$\chi^{2}$ & $137,637 * *$ & & 52,778 & & $74,061 * * *$ & \\
\hline df & 82 & & 53 & & 40 & \\
\hline$\chi^{2} / \mathrm{df}$ & 1,68 & & 1,00 & & 1,85 & \\
\hline CFI & 0,914 & & 1,000 & & 0,888 & \\
\hline RMSEA & 0,056 & & 0,000 & & 0,085 & \\
\hline SRMR & 0,049 & & 0,040 & & 0,058 & \\
\hline $\mathrm{AIC}$ & 9207,177 & & 4360,623 & & 4046,240 & \\
\hline
\end{tabular}

Anmerkung: *p<0,05, **p<0,01, ***p $<0,001$

Kodierung der Variable Geschlecht: $0=$ weiblich, 1 = männlich; Kodierung des Leistungskurses Deutsch (Modell 1), Englisch (Modell 2), Mathematik (Modell 3) in der Sekundarstufe II: 0 = nicht besucht, $1=$ besucht

CFI Comparative Fit Index, RMSEA Root Mean Square Error of Approximation, SRMR Standardized Root Mean Square Residual, AIC Akaike Information Criterion

vorhersagen können, während dies bei den deutschdidaktischen $(0,14$, n. s.) und den englischdidaktischen Inhalten nicht der Fall ist $(0,14$, n. s.).

Eine differenzierte Betrachtung nach Subskalen der fachdidaktischen Inhalte enthält Tab. 7: Die zuvor in Tab. 6 dargestellten Strukturmodelle wurden jeweils erneut berechnet, jedoch statt der Gesamtskala von Ausbildungsinhalten nun lediglich mit der einzelnen Subskala als Prädiktor (vgl. Tab. 2 für die Kennwerte der Subskalen), sodass Einblicke in die Bedeutung der einzelnen Bereiche von Ausbildungsinhalten gegeben werden können.

Die deutschdidaktischen Inhalte des Schreibens $(0,20)$ und der Sprachreflexion $(0,16)$ sind ersichtlich trennschärfer $(p<0,05)$ bei der spezifischen Vorhersa- 
Tab. 7 Pfadkoeffizienten für einzelne Skalen zur Erfassung fachdidaktischer Ausbildungsinhalte, resultierend aus einer Serie von Pfadmodellen

\begin{tabular}{|c|c|c|c|c|c|c|c|c|}
\hline \multirow[t]{2}{*}{$\begin{array}{l}\text { Fach- } \\
\text { didaktik }\end{array}$} & \multirow[t]{2}{*}{ Subskala } & \multirow[t]{2}{*}{ Modell } & \multicolumn{2}{|c|}{$\begin{array}{l}\text { Pädagogisches } \\
\text { Wissen }\end{array}$} & \multirow[b]{2}{*}{$p$} & \multicolumn{3}{|c|}{$\begin{array}{l}\text { Fachdidaktisches } \\
\text { Wissen }\end{array}$} \\
\hline & & & $\beta$ & SE & & $\beta$ & SE & $p$ \\
\hline \multirow[t]{6}{*}{ Deutsch } & $\begin{array}{l}\text { Sprechen und Zuhö- } \\
\text { ren }\end{array}$ & 1 & 0,21 & 0,06 & $<0,01$ & 0,21 & 0,09 & $<0,05$ \\
\hline & Schreiben & 2 & 0,08 & 0,06 & n.s. & 0,20 & 0,09 & $<0,05$ \\
\hline & Lesen & 3 & 0,06 & 0,06 & n.s. & $-0,08$ & 0,09 & n.s. \\
\hline & $\begin{array}{l}\text { Lesen literarischer } \\
\text { Texte }\end{array}$ & 4 & 0,07 & 0,06 & n. s. & $-0,01$ & 0,08 & n.s. \\
\hline & Sprachreflexion & 5 & 0,06 & 0,05 & n.s. & 0,16 & 0,07 & $<0,05$ \\
\hline & Curriculare Aspekte & 6 & 0,01 & 0,06 & n. s. & 0,11 & 0,09 & n. s. \\
\hline \multirow[t]{7}{*}{ Englisch } & Sprachdidaktik & 7 & 0,09 & 0,09 & n.s. & 0,29 & 0,09 & $<0,01$ \\
\hline & Literaturdidaktik & 8 & 0,16 & 0,08 & $<0,05$ & 0,06 & 0,09 & n.s. \\
\hline & Kulturdidaktik & 9 & 0,07 & 0,08 & n. s. & 0,31 & 0,09 & $<0,001$ \\
\hline & Mediendidaktik & 10 & 0,17 & 0,07 & $<0,05$ & 0,20 & 0,08 & $<0,01$ \\
\hline & Methodik & 11 & 0,05 & 0,09 & n.s. & 0,12 & 0,09 & n.s. \\
\hline & $\begin{array}{l}\text { Curriculum/ } \\
\text { Beurteilung }\end{array}$ & 12 & 0,20 & 0,09 & $<0,05$ & 0,29 & 0,09 & $<0,01$ \\
\hline & $\begin{array}{l}\text { Fachdidaktische } \\
\text { Forschung }\end{array}$ & 13 & 0,04 & 0,09 & n.s. & 0,27 & 0,10 & $<0,01$ \\
\hline \multirow{6}{*}{$\begin{array}{l}\text { Mathe- } \\
\text { Matik }\end{array}$} & Grundlagen & 14 & 0,17 & 0,11 & n. s. & 0,01 & 0,13 & n.s. \\
\hline & Heterogenität & 15 & 0,09 & 0,11 & n.s. & 0,03 & 0,13 & n.s. \\
\hline & Technologie & 16 & 0,16 & 0,08 & n. s. & $-0,07$ & 0,10 & n.s. \\
\hline & $\begin{array}{l}\text { Methodik/ } \\
\text { Unterrichtspraxis }\end{array}$ & 17 & 0,13 & 0,11 & n. s. & 0,08 & 0,13 & n.s. \\
\hline & Curriculum & 18 & 0,16 & 0,10 & n. s. & 0,08 & 0,12 & n.s. \\
\hline & $\begin{array}{l}\text { Fachdidaktische } \\
\text { Forschung }\end{array}$ & 19 & 0,20 & 0,09 & $<0,05$ & 0,24 & 0,10 & $<0,05$ \\
\hline
\end{tabular}

ge deutschdidaktischen Wissens im Vergleich zum pädagogischen Wissen (jeweils n. s.). Der Inhalt „Sprechen und Zuhören“ kann in ähnlicher Höhe das deutschdidaktische wie das pädagogische Wissen statistisch signifikant vorhersagen $(0,21)$. Das englischdidaktische Wissen kann statistisch signifikant durch die Prädiktoren aus den Bereichen Kulturdidaktik ( 0,31$)$, Sprachdidaktik $(0,29)$, Curriculum/Beurteilung $(0,29)$, fachdidaktische Forschung $(0,27)$, und Mediendidaktik $(0,20)$ erklärt werden, nicht signifikant sind die Prädiktoren Literaturdidaktik und Methodik. Für das pädagogische Wissen sind die Inhalte Curriculum/Beurteilung $(0,20)$, Mediendidaktik $(0,17)$ und Literaturdidaktik $(0,16)$ als Prädiktoren signifikant. Insgesamt sind also die Inhalte der Englischdidaktik eher trennscharf bei der spezifischen Vorhersage englischdidaktischen Wissens im Vergleich zum pädagogischen Wissen. Im Bereich der Mathematikdidaktik fallen dagegen die Prädiktoren nicht signifikant aus, mit Ausnahme des Prädiktors der fachdidaktischen Forschung $(0,24)$, der jedoch auch zugleich das pädagogische Wissen erklären kann $(0,20)$. 


\section{Diskussion}

\subsection{Zusammenfassung}

Pädagogisches und fachdidaktisches Wissen gehören zusammen mit dem fachlichen Wissen zweifelsfrei zum Kern der professionellen Kompetenz von Lehrkräften. Auf einer übergreifenden Ebene finden sich relativ konsensfähige Beschreibungen dieser Wissensbereiche, etwa die Abgrenzung des Fachwissens (CK), das von den akademischen Fachdisziplinen geprägt ist, vom pädagogischen Wissen (GPK), das ein breites Spektrum generischer Aspekte von der Unterrichtsmethodik und Klassenführung bis hin zur Diagnostik und Leistungsbeurteilung umfasst, sowie die vielfach genannte Funktion des fachdidaktischen Wissens (PCK), Fachinhalte unter der Perspektive ihrer Lehr- und Lernbarkeit in schulischen Situationen zu betrachten. Auf einer differenzierten Ebene kann die Abgrenzung zwischen pädagogischem und fachdidaktischem Wissen jedoch Schwierigkeiten mit sich bringen, zum Beispiel bei der Frage, inwieweit für die Berücksichtigung kognitiver Lernvoraussetzungen der Schülerinnen und Schüler bei der Unterrichtsgestaltung allein Wissen über allgemeine Prinzipien der Unterrichtsgestaltung ausreichend oder auch Wissen über domänenspezifische Konkretisierungen vonnöten ist.

In der universitären Lehramtsausbildung werden angehende Lehrkräfte beim Erwerb pädagogischen und fachdidaktischen Wissens unterstützt, typischerweise sind die Zuständigkeiten jedoch institutionell getrennt (Blömeke 2002). Hinzu kommt, dass Lehramtsstudierende als Novizen ihr Wissen zunächst nach den einzelnen Anforderungsbereichen separiert erwerben und erst später, im Zuge der Reflexion ihrer Erfahrungen zunehmend vernetzen (u. a. Berliner 2004; Clift und Brady 2005; Flores 2016). Vor diesem Hintergrund gingen wir im vorliegenden Beitrag zwei Fragestellungen nach für die Bearbeitung, einer zur Struktur pädagogischen Wissens und fachdidaktischen Wissens in Deutsch, Englisch und Mathematik bei Lehramtsstudierenden von zwei Volluniversitäten in Deutschland und, darauf aufbauend, einer zur Validität der dabei verwendeten Wissenstests.

Hinsichtlich der Struktur von pädagogischem und fachdidaktischem Wissen hatten wir vermutet, dass bei der Zielgruppe angehender Lehrkräfte der ersten Ausbildungsphase pädagogisches und fachdidaktisches Wissen empirisch getrennt werden können. Ferner hatten wir erwartet, dass pädagogisches Wissen und Wissen in den sprachlichen Domänen angesichts des hier vermutlich stärker ausgeprägten narrativen Charakters des Wissens höher korreliert sind als pädagogisches und mathematikdidaktisches Wissen. Im Hinblick auf die Validität hatten wir vermutet, dass Master-Studierende angesichts ihres fortgeschrittenen Ausbildungsstadiums bei der Testung pädagogischen und fachdidaktischen Wissens besser abschneiden würden als Bachelor-Studierende; Inhalte der fachdidaktischen Ausbildung, so unsere weitere Vermutung, sollten zudem als Prädiktor Testleistungen im fachdidaktischen, jedoch nicht unbedingt im pädagogischen Wissen erklären können.

Ergebnisse aus ein- und zweidimensionalen Faktorenanalysen zeigten für jede der drei Domänen Deutsch, Englisch und Mathematik, dass eine empirische Trennung in die beiden Wissensbereiche einer eindimensionalen Modellierung überlegen ist; unterschiedlich hohe Interkorrelationen der sprachlichen Fächer gegenüber der 
Mathematik zeigten sich dabei (zunächst) nicht. Wie erwartet erreichten die fortgeschrittenen Master-Studierenden bessere Testleistungen als die Bachelor-Studierenden, wobei zumindest für das deutschdidaktische und das englischdidaktische Wissen spezifische Ausbildungsinhalte als Prädiktor zusätzlich Varianz in der fachdidaktischen Testung aufklären konnten.

\subsection{Diskussion der Befunde}

Zunächst sprechen die Ergebnisse grundsätzlich für die vermutete Differenzierung des professionellen Wissens bei Lehramtsstudierenden in pädagogisches und fachdidaktisches Wissen. Neben den Analysen zur ersten Fragestellung (Tab. 5) ist den Pfadmodellen zur zweiten Fragestellung (Tab. 6) zu entnehmen, dass bei Kontrolle weiterer Variablen die Interkorrelationen sogar zurückgehen (von 0,55/0,65/0,64 auf 0,33/0,48/0,25). Mit Blick auf die inferenzstatistische Absicherung betrifft der Rückgang vor allem die Korrelation des pädagogischen mit dem mathematikdidaktischen Wissen, welche im betreffenden Pfadmodell nicht mehr statistisch signifikant ist (Tab. 6, Modell 3). Dieses Ergebnis unterscheidet sich von dem in den sprachlichen Domänen, was unserer aufgestellten Hypothese (H1b) entspricht.

Damit schließen unsere Analysen an bisherige Strukturanalysen zum professionellen Wissen von angehenden und berufstätigen Lehrkräften an (vgl. Abschn. 2.3.2). Gegenüber den Strukturanalysen in TEDS-LT (König et al. 2011) besteht der Mehrwert der vorliegenden Analyse unter anderem in der Modellierung auf Ebene latenter Variablen sowie in der Verwendung von Stichproben, die hinsichtlich ihres Fachsemesters klar definiert sind (wie wir nachfolgend erneut aufgreifen werden). Die Ergebnisse unserer Analysen leisten einen Beitrag zur Diskussion um die angenommene Differenzierung des professionellen Lehrerwissens in den Kategorien des fachdidaktischen und pädagogischen Wissens (Shulman 1987; Baumert und Kunter 2006).

Die Wissensunterschiede zwischen Bachelor- und Masterstudierenden können einerseits als Beleg für curriculare Validität der verwendeten Tests angesehen werden, andererseits ist zu konstatieren, dass entgegen häufig geäußerter Kritik an der universitären Lehrerbildung diese den Erwerb professionellen Lehrerwissens offenbar doch erheblich unterstützt. Zwar beruhen die dargelegten Wissensunterschiede lediglich auf Kohortenvergleichen, sie liefern aber Hinweise auf die Wirksamkeit der Ausbildung in den einbezogenen Lehramtsstudiengängen beider Universitäten, welche in zukünftigen Längsschnittstichproben abgesichert werden sollten. Im Falle des pädagogischen (König und Klemenz 2015) und mathematikdidaktischen Wissens (Buchholtz et al. 2011, 2013) liegen einschlägige Ergebnisse zu den verwendeten Tests bereits vor. Sie wären um Längsschnittstudien zu ergänzen, die die Tests zur Erfassung von deutsch- und englischdidaktischem Wissen einsetzen.

Die hier berichteten Befunde zu Unterschieden im Wissen in Abhängigkeit des Ausbildungsstadiums schließen somit an die Ergebnisse anderer Studien an. Vor allem für mathematikdidaktisches Wissen (neben TEDS-M und Studien im Kontext von TEDS-M vor allem die COACTIV-Studie, vgl. Kleickmann et al. 2013) oder fachdidaktisches Wissen in naturwissenschaftlichen Fächern (z. B. für Physikdidaktik, vgl. Riese und Reinhold 2012) konnten bislang ähnliche Unterschiede zwi- 
schen Gruppen von Lehramtsstudierenden in unterschiedlichen Ausbildungsstadien erbracht werden, die für einen Erwerb fachdidaktischen Wissens während des universitären Studiums sprechen. Für sprachliche Fächer ist der Forschungsstand hingegen noch sehr dünn, sodass die hier erbrachten Ergebnisse einen deutlichen Erkenntnisgewinn darstellen dürften. Von Bedeutung sind zwar die Analysen aus TEDS-LT zur Abschätzung von Wissensunterschieden zwischen Bachelor- und Masterstudierenden der Fächer Deutsch, Englisch und Mathematik, die bereits auf kleine Wissensvorsprünge der Master- gegenüber den Bachelor-Studierenden verweisen konnten (Blömeke 2013). Allerdings enthalten die in TEDS-LT verwendeten Stichproben Studierende aus sehr unterschiedlichen Fachsemestern (Stancel-Piątak et al. 2013, S. 41), die eine eindeutige Definition von Ausbildungsstadien erschweren und damit die Aussagekraft einschränken. Dies könnte erklären, warum in der vorliegenden Studie auf Basis klar definierter Stichproben (jeweiliges 2. Semester im Bachelorund Master-Studiengang) stärkere Unterschiede in Abhängigkeit des Ausbildungsstadiums festgestellt werden konnten (Tab. 6) als es in TEDS-LT möglich war. Eine andere Erklärung könnte sein, dass es sich im Falle der deutschdidaktischen und englischdidaktischen Wissenstests nicht um identische, sondern um weiterentwickelte Instrumente handelt, die hinsichtlich des Ausbildungsstadiums sensitiver als die Instrumente aus TEDS-LT sind.

Dass zumindest für das deutschdidaktische und das englischdidaktische Wissen spezifische Ausbildungsinhalte als Prädiktor zusätzlich Varianz in der fachdidaktischen Testung aufklären konnten, korrespondiert mit dem grundsätzlichen Ziel der Lehrerausbildung, den Erwerb fachdidaktischen Wissens von Lehrpersonen durch domänenspezifische Lerngelegenheiten zu unterstützen. Entgegen unserer Erwartung konnten die Ausbildungsinhalte in der Mathematikdidaktik mit Ausnahme der Subskala „fachdidaktische Forschung“ als Prädiktor die Testleistungen allerdings nicht zusätzlich erklären (Tab. 7). Hier bietet sich möglicherweise als Erklärung an, dass das mathematikdidaktische Curriculum einen höheren Strukturierungsgrad aufweist als die Curricula in sprachlichen Fächern des Lehramtsstudiums. Die standortübergreifend stärkere Kanonisierung der Ausbildungsinhalte und eine damit einhergehende geringere Wahlfreiheit im beobachteten Studienabschnitt (zwischen 2. Bachelorund 2. Master-Semester) könnte zu geringeren inter-individuellen Schwerpunktsetzungen im Hinblick auf Ausbildungsinhalte beitragen, was sich bei unserer Analyse in Form von schwachen Prädiktoren niederschlägt. Sprachliche Fächer hingegen bieten mehr Möglichkeiten eigener Schwerpunktbildungen, sodass sie - in entsprechenden Bereichen - inter-individuelle Unterschiede im Studierverhalten abbilden können. Dies könnte auch als mögliche Erklärung dienen, warum der für die Englischdidaktik wichtige Inhalt „Methodik“ als Prädiktor nicht signifikant ist, obgleich ansonsten englischdidaktisches Wissen recht gut durch fachdidaktische Inhalte erklärt werden kann (vgl. auch König et al. 2017). Außerdem ist denkbar, dass ein höherer Strukturierungsgrad der vorgegebenen Ausbildungsinhalte in der Mathematikdidaktik zwar weniger eigene Schwerpunktsetzungen innerhalb eines Semesters erlaubt, sich aber in stärkeren Unterschieden in den Testleistungen nach Ausbildungsstadium (Bachelor vs. Master) bemerkbar macht. Wie anhand der Kennwerte für den Studienabschnitt als Prädiktor in Tab. 6 ersichtlich ist, fallen die Unterschiede zwischen den beiden betrachteten Kohorten in der mathematikdidaktischen Testung 
tatsächlich größer aus $(0,51)$ als in der deutschdidaktischen $(0,28)$ und englischdidaktischen $(0,30)$. Schließlich wäre zu berücksichtigen, dass, wie zuvor dargestellt (vgl. Abschn. 4.2.3), der mathematikdidaktische Wissenstest (wie auch der englischdidaktische, nicht aber der deutschdidaktische Wissenstest) anforderungsbezogen konzipiert ist, die Instrumente zur Erfassung der fachdidaktischen Ausbildungsinhalte hingegen durchgehend auf Basis des Curriculums entwickelt wurden. Ein solcher Unterschied in der Konzeptualisierung kann möglicherweise dazu führen, dass eine Vorhersage des Wissens durch die Lerngelegenheiten erschwert wird.

Ebenfalls entgegen unserer Erwartung konnte das pädagogische Wissen vereinzelt durch spezifische fachdidaktische Ausbildungsinhalte vorhergesagt werden. Dies ist in allen drei Domänen vereinzelt beobachtbar (Deutschdidaktik: Sprechen und Zuhören; Englischdidaktik: Literatur- und Mediendidaktik, Curriculum/Beurteilung; Mathematikdidaktik: Fachdidaktische Forschung, vgl. Tab. 7). Zumindest die folgenden Erklärungen sind denkbar. Es liegen möglicherweise Überschneidungen zwischen einzelnen fachdidaktischen Ausbildungsinhalten und den Inhalten des getesteten pädagogischen Wissens vor (z. B. ,Beurteilung“ als englischdidaktischer Inhalt und „Leistungsbeurteilung“ als Inhalt des Tests zum pädagogischen Wissen, vgl. Abb. 4). Ferner ist es möglich, dass die gemessenen Ausbildungsinhalte Studienverlaufsinformationen enthalten, die anteilig mit den erreichten Testleistungen korrespondieren. Auch könnten sich Messfehler bemerkbar machen (z. B. bei der Subskala „Sprechen und Zuhören“, vgl. Tab. 2). Diese Ungenauigkeiten sind kritisch zu betrachten und machen vertiefende Analysen in zukünftigen Untersuchungen erforderlich.

\subsection{Limitationen und Ausblick auf zukünftige Forschung}

Dass das Design der Studie nicht echt, sondern nur quasi-längsschnittlich ist, wurde bereits erwähnt. Zwei weitere Limitationen unserer Studie seien noch angesprochen. Aus forschungsökonomischen Gründen konnte fachliches Wissen der Lehramtsstudierenden nicht in die vorliegende Analyse einbezogen werden. Dies wäre jedoch ein weiterer wichtiger Analyseschritt, um das Verhältnis zwischen pädagogischem und fachdidaktischem Wissen noch präziser, als es hier möglich war, in den Blick zu nehmen (vgl. König et al. 2016). Zumindest an einer der beiden einbezogenen Universitäten wurde das fachliche Wissen ebenfalls getestet, sodass mit weiteren Analysen in naher Zukunft zu rechnen ist. Eine weitere Beschränkung betrifft die Modellierung der Wirkungen von Lerngelegenheiten auf den vermuteten Zuwachs von Wissen. In diesem Bereich liegen bislang nur vereinzelt Studien vor, die methodisch anspruchsvoller angelegt sind, etwa mit echten Längsschnittstichproben und unter Einbezug weiterer Indikatoren wie der von den Studierenden wahrgenommenen Prozessqualität. Zukünftige Studien könnten sich hier der Herausforderung stellen, Ansätze des Angebots-Nutzungs-Konzepts aus der Schul- und Unterrichtsforschung auf die Hochschulforschung zu übertragen wie es für empirische Studien zum pädagogischen Wissen bereits erfolgt ist (König und Seifert 2012). Es wäre wünschenswert, wenn diese und weitere Desiderata von aktuellen Initiativen wie den Projekten der von Bund und Ländern geförderten „Qualitätsoffensive Lehrerbildung“ aufgegriffen werden würden. So könnten in naher Zukunft deutliche 
Fortschritte in der Modellierung des Erwerbs pädagogischen und fachdidaktischen Wissens angehender Lehrkräfte erzielt werden.

\subsection{Implikationen und Fazit}

Unsere Studie stärkt für die drei Domänen Deutsch, Englisch und Mathematik die Annahme, dass Lehramtsstudierende fachdidaktisches und pädagogisches Wissen während ihrer universitären Ausbildung erwerben. Ihr fachdidaktisches Wissen und ihr pädagogisches Wissen lassen sich empirisch über Wissenstests erfassen und dabei relativ eindeutig als eigene Wissenskategorien unterscheiden. Fachdidaktische Inhalte des Ausbildungscurriculums können für den Erwerb fachdidaktischen Wissens eine Bedeutung haben, der Nachweis dafür ist allerdings methodisch nicht einfach zu erbringen. Es bedarf weiterer Forschung, um die zugrundeliegenden Annahmen zur Wirksamkeit der Lehrerbildung zu prüfen.

Open Access Dieser Artikel wird unter der Creative Commons Namensnennung 4.0 International Lizenz (http://creativecommons.org/licenses/by/4.0/deed.de) veröffentlicht, welche die Nutzung, Vervielfältigung, Bearbeitung, Verbreitung und Wiedergabe in jeglichem Medium und Format erlaubt, sofern Sie den/die ursprünglichen Autor(en) und die Quelle ordnungsgemäß nennen, einen Link zur Creative Commons Lizenz beifügen und angeben, ob Änderungen vorgenommen wurden.

\section{Literatur}

Anderson, L. W., \& Krathwohl, D. R. (Hrsg.) (2001). A taxonomy for learning, teaching, and assessing: A revision of bloom's taxonomy of educational objectives. New York: Longman.

Ball, D. L., Thames, M.H., \& Phelps, G. (2008). Content knowledge for teaching: What makes it special? Journal of Teacher Education, 59, 389-407.

Bandalos, D. L., \& Finney, S. J. (2009). Item parceling issues in structural equation modeling. In G. A. Marcoulides \& R. E. Schumacker (Hrsg.), New developments and techniques in structural equation modeling (S. 269-296). Mahwah: Lawrence Erlbaum.

Baumert, J. (2016). Leistungen, Leistungsfähigkeit und Leistungsgrenzen der empirischen Bildungsforschung: Das Beispiel von Large-Scale-Assessment-Studien zwischen Wissenschaft und Politik. Zeitschrift für Erziehungswissenschaft, 19(1), 215-253.

Baumert, J., \& Kunter, M. (2006). Stichwort: Professionelle Kompetenz von Lehrkräften. Zeitschrift für Erziehungswissenschaft, 9(4), 469-520.

Baumert, J., Kunter, M., Blum, W., Brunner, M., Voss, T., Jordan, A., Klusmann, U., Krauss, S., Neubrand, M., \& Tsai, Y.-M. (2010). Teachers' mathematical knowledge, cognitive activation in the classroom, and student progress. American Educational Research Journal, 47, 133-180.

Berliner, D.C. (2004). Describing the behavior and documenting the accomplishments of expert teachers. Bulletin of Science, Technology \& Society, 24, 200-212.

Blömeke, S. (2002). Universität und Lehrerausbildung. Bad Heilbrunn: Klinkhardt.

Blömeke, S. (2004). Empirische Befunde zur Wirksamkeit der Lehrerbildung. In S. Blömeke, P. Reinhold, G. Tulodziecki, \& J. Wildt (Hrsg.), Handbuch Lehrerbildung (S. 59-91). Bad Heilbrunn: Klinkhardt.

Blömeke, S. (2013). Einleitung: Professionelle Kompetenzen im Studienverlauf. In S. Blömeke, A. Bremerich-Vos, G. Kaiser, G. Nold, H. Haudeck, J.-U. Keßler, \& K. Schwippert (Hrsg.), Professionelle Kompetenzen im Studienverlauf. Weitere Ergebnisse zur Deutsch-, Englisch- und Mathematiklehrerausbildung aus TEDS-LT (S. 7-24). Münster: Waxmann.

Blömeke, S., \& Delaney, S. (2012). Assessment of teacher knowledge across countries: A review of the state of research. ZDM, 44(3), 223-247.

Blömeke, S., Herzig, B., \& Tulodziecki, G. (2007). Gestaltung von Schule. Eine Einführung in Schultheorie und Schulentwicklung. Bad Heilbrunn: Klinkhardt.

Blömeke, S., Kaiser, G., \& Lehmann, R. (Hrsg.) (2010). TEDS-M 2008. Professionelle Kompetenz und Lerngelegenheiten angehender Mathematiklehrkräfte für die Sekundarstufe I im internationalen Vergleich. Münster: Waxmann Verlag. 
Blömeke, S., Bremerich-Vos, A., Haudeck, H., Kaiser, G., Lehmann, R., Nold, G., Schwippert, K., \& Willenberg, H. (Hrsg.) (2011). Kompetenzen von Lehramtsstudierenden in gering strukturierten Domänen. Erste Ergebnisse aus TEDS-LT. Münster: Waxmann.

Blömeke, S., Gustafsson, J.-E., \& Shavelson, R. (2015). Beyond dichotomies: Competence viewed as a continuum. Zeitschrift für Psychologie, 223(1), 3-13.

Bond, T., \& Fox, C. (2007). Applying the Rasch model: Fundamental measurement in the human sciences (2. Aufl.). Mahwah, NJ: Lawrence Erlbaum.

Borg, S. (2006). The distinctive characteristics of foreign language teachers. Language Teaching Research, $10,3-31$.

Bremerich-Vos, A., \& Dämmer, J. (2013). Professionelles Wissen im Studienverlauf: Lehramt Deutsch. In S. Blömeke, A. Bremerich-Vos, G. Kaiser, G. Nold, H. Haudeck, J.-U. Keßler, \& K. Schwippert (Hrsg.), Professionelle Kompetenzen im Studienverlauf. Weitere Ergebnisse zur Deutsch-, Englischund Mathematiklehrerausbildung aus TEDS-LT (S. 47-75). Münster: Waxmann.

Bremerich-Vos, A. (2017). Adaptives Lehren im Literaturunterricht? Erscheint in: D. Wrobel, T. von Brand, \& M. Engelns (Hrsg.), Gestaltungsraum Deutschunterricht. Literatur - Kultur - Sprache. Hohengehren: Schneider.

Bromme, R. (1992). Der Lehrer als Experte. Bern: Huber.

Bromme, R. (2001). Teacher Expertise. In International Encyclopedia of the Social \& Behavioral Sciences (S. 15459-15465). Amsterdam: Smelser and Baltes.

Bruner, J. S. (1986). Actual minds. Possible worlds. Cambridge: Harvard University Press.

Bruner, J. S. (1991). The narrative construction of reality. Critical Inquiry, 18(1), 1-21.

Buchholtz, N., \& Kaiser, G. (2013). Improving mathematics teacher education in Germany: Empirical results from a longitudinal evaluation. International Journal of Science and Mathematics Education, 11(4), 949-977.

Buchholtz, N., Blömeke, S., Kaiser, G., König, J., Lehmann, R., Schwarz, B., \& Suhl, U. (2011). Entwicklung von Professionswissen im Lehramtsstudium: eine Längsschnittstudie an fünf deutschen Universitäten. In K. Eilerts, A. Hilligus, G. Kaiser, \& P. Bender (Hrsg.), Kompetenzorientierung in Schule und Lehrerbildung (S. 201-214). Paderborn: Lit Verlag.

Buchholtz, N., Scheiner, T., Döhrmann, M., Suhl, U., Kaiser, G., \& Blömeke, S. (2016). TEDS-shortM. Kurzfassung der mathematischen und mathematikdidaktischen Testinstrumente aus TEDS-M, TEDSLT und TEDS-Telekom. Hamburg: Universität Hamburg.

Bukova-Güzel, E. (2010). An investigation of pre-service mathematics teachers' pedagogical content knowledge, using solid objects. Scientific Research and Essays, 5, 1872-1880.

BMBF (2014) = Bundesministerium für Bildung und Forschung. (2014). Bekanntmachung des Bundesministeriums für Bildung und Forschung von Richtlinien zur Förderung der „Qualitätsoffensive Lehrerbildung “. Berlin: BMBF.

Burns, A., Freeman, D., \& Edwards, E. (2015). Theorizing and studying the language-teaching mind: Mapping research on language teacher cognition. Modern Language Journal, 99, 585-601.

Clandinin, D. J. (1992). Narrative and story in teacher education. In T. Russell \& H. Munby (Hrsg.), Teachers and eaching: From classroom to reflection (S. 124-137). London: Falmer Press.

Clift, R. T., \& Brady, P. (2005). Research on methods courses and field experiences. In M. Cochran-Smith \& K. M. Zeichner (Hrsg.), Studying teacher education. The report of the AERA panel on research and teacher education (S. 309-424). Mahwah, NJ: Lawrence Erlbaum.

Depaepe, F., Verschaffel, L., \& Kelchtermans, G. (2013). Pedagogical content knowledge: A systematic review of the way in which the concept has pervaded mathematics educational research. Teaching and Teacher Education, 34, 12-25.

DMV, GDM \& MNU (2008) = Deutsche Mathematiker-Vereinigung. Gesellschaft für Didaktik der Mathematik. \& Deutscher Verein zur Förderung des mathematischen und naturwissenschaftlichen Unterrichts (2008). Standards für die Lehrerbildung im Fach Mathematik. Empfehlungen von DMV, GDM, MNU.

Eurostat. (2012). Eurostat news release 144/2014. Luxembourg: Eurostat Press Office.

Expertenkommission (2007). Ausbildung von Lehrern in NRW. Düsseldorf: MSW.

Fend, H. (2008). Schule gestalten. Systemsteuerung, Schulentwicklung und Unterrichtsqualität. Wiesbaden: Springer VS.

Fenstermacher, G. D. (1994). The knower and the known: The nature of knowledge in research on teaching. In L. Darling Hammond (Hrsg.), Review of research in education (Bd. 20, S. 3-56). Washington, DC: American Educational Research Association. 
Floden, R. (2015). Learning what research says about teacher preparation. In M. J. Feuer, A. I. Berman, \& R. C. Atkinson (Hrsg.), Past as prologue: The national academy of education at 50: Members reflect (S. 279-284). Washington, DC: National Academy of Education.

Flores, M. A. (2016). Teacher education curriculum. In J. Loughran \& M. L. Hamilton (Hrsg.), International handbook of teacher education (S. 187-230). Dordrecht, the Netherlands: Springer.

Freeman, D. (2002). The hidden side of the work: teacher knowledge and learning to teach. Language Testing, 35, 1-13.

Gatbonton, E. (1999). Investigating experienced ESL teachers' pedagogical knowledge. The Modern Language Journal, 83(1), 35-50.

Gitomer, D.H., \& Zisk, R.C. (2015). Knowing what teachers know. Review of Research in Education, 39, $1-53$.

Grossman, P.L. (1990). The making of a teacher: Teacher knowledge and teacher education. New York: Teachers College Press.

Grossman, P. L., \& Shulman, L. S. (1994). Knowing, believing, and the teaching of English. In T. Shanahan (Hrsg.), Teachers thinking, teachers knowing: Reflections on literacy and language education (S. 3-22). Urbana, IL: National Council of Teachers of English.

Großschedl, J., Harms, U., Kleickmann, T., \& Glowinski, I. (2015). Preservice biology teachers' professional knowledge: Structure and learning opportunities. Journal of Science Teacher Education, 26(3), 291-318.

Helmke, A. (2012). Unterrichtsqualität und Lehrerprofessionalität: Diagnose, Evaluafion und Verbesserung des Unterrichts (4., überarb. Aufl.). Seelze: Klett-Kallmeyer.

Hill, H.C., Rowan, B., \& Ball, D.L. (2005). Effects of teachers' mathematical knowledge for teaching on student achievement. American Educational Research Journal, 42, 371-406.

Hinkel, E. (2006). Current perspectives on teaching the four skills. Tesol Quarterly, 40(1), 109-131.

Hogan, T., Rabinowitz, M., \& Craven III, J. A. (2003). Representation in teaching: Inferences from research of expert and novice teachers. Educational Psychologist, 38, 235-247.

House, J. (2003). English as a lingua franca: A threat to multilingualism? Journal of Sociolinguistics, 7(4), $556-578$.

Hu, L., \& Bentler, P. M. (1999). Cutoff criteria for fit index in covariance structure analysis: Conventional criteria versus new alternatives. Structural Equation Modeling. A Multidisciplinary Journal, 6, 1-55.

Jansing, B., Haudeck, H., Keßler, J.-U., Nold, G., \& Stancel-Piątak, A. (2013). Professionelles Wissen im Studienverlauf: Lehramt Englisch. In S. Blömeke, A. Bremerich-Vos, G. Kaiser, G. Nold, H. Haudeck, J.-U. Keßler, \& K. Schwippert (Hrsg.), Professionelle Kompetenzen im Studienverlauf. Weitere Ergebnisse zur Deutsch-, Englisch- und Mathematiklehrerausbildung aus TEDS-LT (S. 77-106). Münster: Waxmann.

Jenkins, J. (2013). English as a Lingua Franca in the international university (The politics of academic English language policy). New York, NY: Routledge.

Kammler, C. (2000). Neue Literaturtheorien und Unterrichtspraxis. Positionen und Modelle. Baltmannsweiler: Schneider Hohengehren.

Kennedy, M. (1990). A survey of recent literature on teachers' subject matter knowledge (research rep. nr. 90-3).

Kleickmann, T., Richter, D., Kunter, M., Elsner, J., Besser, M., Krauss, S., \& Baumert, J. (2013). Teachers' content knowledge and pedagogical content knowledge: The role of structural differences in teacher education. Journal of Teacher Education, 64(1), 90-106.

Kline, R. B. (2005). Principles and practice of structural equation modelling. New York: Guilford.

KMK (2004/2014) = Kultusministerkonferenz. (2004/2014). Standards für die Lehrerbildung: Bildungswissenschaften. Beschluss der Kultusministerkonferenz. Bonn: KMK.

KMK (2008) = Kultusministerkonferenz. (2008). Ländergemeinsame inhaltiche Anforderungen für die Fachwissenschaften und Fachdidaktiken in der Lehrerinnen- und Lehrerbildung (Beschluss der Kultusministerkonferenz vom 16. Oktober 2008). Bonn: KMK.

KMK (2009) = Kultusministerkonferenz. (2009). Information des Sekretariats über die Regelungen des KMK-Beschlusses vom 22.10.1999 'Gegenseitige Anerkennung von Lehramtsprüfungen und Lehramtsbefähigungen' (Stand: 05. Februar 2009). Bonn: KMK.

KMK (2012) = Kultusministerkonferenz. (2012). Bildungsstandards für die fortgeführte Fremdsprache (Englisch/Französisch) für die Allgemeine Hochschulreife (Beschluss der Kultusministerkonferenz vom 18. Oktober 2012). Bonn: KMK.

König, J. (2014). Designing an international instrument to assess teachers' general pedagogical knowledge (GPK): Review of studies, considerations, and recommendations. Technical paper prepared for the OECD innovative teaching for effective learning (ITEL) - phase II project: A survey to profile the 
pedagogical knowledge in the teaching profession (ITEL teacher knowledge survey). http://www. oecd.org/officialdocuments/publicdisplaydocumentpdf/?cote=EDU/CERI/CD/RD\%282014\%293/ REV1\&doclanguage=en. Zugegriffen: 12. Juni 2017.

König, J., \& Blömeke, S. (2009). Pädagogisches Wissen von angehenden Lehrkräften: Erfassung und Struktur von Ergebnissen der fachübergreifenden Lehrerausbildung. Zeitschrift für Erziehungswissenschaft, 12(3), 499-527.

König, J., \& Blömeke, S. (2010). Pädagogisches Unterrichtswissen. Dokumentation der Kurzfassung des TEDS-M-Testinstruments zur Kompetenzmessung in der ersten Phase der Lehrerausbildung. Berlin: Humboldt-Universität.

König, J., \& Klemenz, S. (2015). Der Erwerb von pädagogischem Wissen bei angehenden Lehrkräften in unterschiedlichen Ausbildungskontexten: Zur Wirksamkeit der Lehrerausbildung in Deutschland und Österreich. Zeitschrift für Erziehungswissenschaft, 18(2), 247-277.

König, J., \& Seifert, A. (Hrsg.) (2012). Lehramtsstudierende erwerben pädagogisches Professionswissen. Ergebnisse der Längsschnittstudie LEK zur Wirksamkeit der erziehungswissenschaftlichen Lehrerausbildung. Münster: Waxmann.

König, J., Blömeke, S., \& Doll, J. (2011). Pädagogisches Wissen von Deutsch-, Englisch- und Mathematiklehramtsstudierenden. In S. Blömeke, A. Bremerich- Vos, H. Haudeck, G. Kaiser, R. Lehmann, G. Nold, K. Schwippert, \& H. Willenberg (Hrsg.), Kompetenzen von Lehramtsstudierenden in gering strukturierten Domänen. Erste Ergebnisse aus TEDS-LT (S. 135-157). Münster: Waxmann.

König, J., Lammerding, S., Nold, G., Rohde, A., Strauß, S., \& Tachtsoglou, S. (2016). Teachers' professional knowledge for teaching english as a foreign language: Assessing the outcomes of teacher education. Journal of Teacher Education, 67(4), 320-337.

König, J., Bremerich-Vos, A., Buchholtz, C., Lammerding, S., Strauß, S., Fladung, I., \& Schleiffer, C. (2017). Die Bedeutung des Professionswissens von Referendarinnen und Referendaren mit Fach Deutsch für ihre Planungskompetenz (PlanvoLL-D). In S. Wernke \& K. Zierer (Hrsg.), Die Unterrichtsplanung: Ein in Vergessenheit geratener Kompetenzbereich?! Status Quo und Perspektiven aus Sicht der empirischen Forschung (S. 121-133). Bad Heilbrunn: Klinkhardt.

König, J., Tachtsoglou, S., Lammerding, S., Strauß, S., Nold, G., \& Rohde, A. (2017). The role of opportunities to learn in teacher preparation for EFL teachers' pedagogical content knowledge. The Modern Language Journal, 101(1), 109-127.

Kunina-Habenicht, O., Lohse-Bossenz, H., Kunter, M., Dicke, T., Förster, D., Gößling, J., Schulze-Stocker, F., Schmeck, A., Baumert, J., Leutner, D., \& Terhart, E. (2012). Welche bildungswissenschaftlichen Inhalte sind wichtig in der Lehrerbildung?. Zeitschrift für Erziehungswissenschaft, 15(4), 649-682.

Kunina-Habenicht, O., Schulze-Stocker, F., Kunter, M., Baumert, J., Leutner, D., Förster, D., Lohse-Bossenz, H., \& Terhart, E. (2013). Die Bedeutung der Lerngelegenheiten im Lehramtsstudium und deren individuelle Nutzung für den Aufbau des bildungswissenschaftlichen Wissens. Zeitschrift für Pädagogik, 59(1), 1-23.

Kunter, M., Baumert, J., Blum, W., Klusmann, U., Krauss, S., \& Neubrand, M. (Hrsg.) (2011). Professionelle Kompetenz von Lehrkräften. Ergebnisse des Forschungsprogramms COACTIV. Münster: Waxmann Verlag.

Lammerding, S., \& König, J. (2017). Modellierung und Erfassung fachdidaktischer Lerngelegenheiten von angehenden Englischlehrkräften: Strukturelle Unterschiede zwischen Universität und Referendariat (Beitrag ist eingereicht).

Lenske, G., Thilmann, H., Wirth, J., Dicke, T., \& Leutner, D. (2015). Pädagogisch-psychologisches Professionswissen von Lehrkräften: Evaluation des ProwiN-Tests. Zeitschrift für Erziehungswissenschaft, $18,225-245$.

Leung, I. K.C., Ding, L., Leung, A. Y.L., \& Wong, N. Y. (2016). The correlation between prospective teachers' knowledge of algebraic inverse operations and teaching competency - using the square root as an example. http://www.cimt.org.uk/ijmtl/index.php/IJMTL/article/download/30/23. Zugegriffen: 12. Juni 2017.

Lipowsky, F. (2006). Auf den Lehrer kommt es an. Empirische Evidenzen für Zusammenhänge zwischen Lehrerkompetenzen, Lehrerhandeln und dem Lernen der Schüler (Zeitschrift für Pädagogik: 51. Beiheft, S. 47-70). Weinheim: Beltz.

Marx, C., Hoehne, V., Voss, T., Goeze, A., Jonkmann, K., Schrader, J., \& Trautwein, U. (2014). Die Verwendung digitaler Medien bei der Erfassung generischer Aspekte des pädagogisch- psychologischen Wissens von Lehrkräften verschiedener Bildungskontexte (Vortrag auf der 2. Nachwuchstagung der Gesellschaft für Empirische Bildungsforschung (GEBF)). Frankfurt a. M.

McDonnell, L. M. (1995). Opportunity to learn as a research concept and a policy instrument. Educational Evaluation and Policy Analysis, 17, 305-322. 
Merzyn, G. (2002). Stimmen zur Lehrerausbildung: Überblick über die Diskussion zur Gymnasiallehrerausbildung, basierend vor allem auf Stellungnahmen von Wissenschafts-und Bildungsgremien sowie auf Erfahrungen von Referendaren und Lehrern. Hohengehren: Schneider.

Morine-Dershimer, G., \& Kent, T. (1999). The complex nature and sources of teachers' pedagogical knowledge. In J. Gess-Newsome \& N. G. Lederman (Hrsg.), Examining pedagogical content knowledge. The construct and its implications for science education (S. 21-50). Netherlands: Springer.

Muthén, L.K., \& Muthén, B.O. (2010). Mplus User's Guide (6. Aufl.). Los Angeles, CA: Muthén \& Muthén.

Oser, F. (2014). Wirkung und Wirkungselemente im Lehrerberuf: Leistungen, Grenzen und Perspektiven aktueller Forschung. In E. Terhart, H. Bennewitz \& M. Rothland (Hrsg.), Handbuch der Forschung zum Lehrerberuf (2. Aufl., S. 764-777). Münster: Waxmann.

Park, S.H., \& Oliver, J.S. (2008). Reconceptualization of pedagogical content knowledge (PCK): PCK as a conceptual tool to understand teachers as professionals. Research in Science Education, 38(3), 261-284.

Praetorius, A. K., Vieluf, S., Saß, S., Bernholt, A., \& Klieme, E. (2016). The same in German as in English? Investigating the subject-specificity of teaching quality. Zeitschrift für Erziehungswissenschaft, 19(1), 191-209.

Reusser, K. (1991). Plädoyer für die Fachdidaktik und für die Ausbildung von Fachdidaktiker/innen für die Lehrerbildung. Beiträge zur Lehrerbildung, 9(2), 193-215.

Riese, J., \& Reinhold, P. (2012). Die professionelle Kompetenz angehender Physiklehrkräfte in verschiedenen Ausbildungsformen. Empirische Hinweise für eine Verbesserung des Lehramtsstudiums. Zeitschrift für Erziehungswissenschaft, 15(1), 111-143.

Schmidt, W.H., Cogan, L., \& Houang, R. (2011). The role of opportunity to learn in teacher preparation: An international context. Journal of Teacher Education, 62, 138-153.

Schön, D. (1983). The reflective practitioner. New York: Basic Books.

Shulman, L.S. (1986). Those who understand: Knowledge growth in teaching. Educational Researcher, 15(2), 4-14.

Shulman, L.S. (1987). Knowledge and teaching: Foundations of the new reform. Harvard Educational Review, 57, 1-22.

Stancel-Piątak, A., Buchholtz, C., \& Schwippert, K. (2013). Anlage und Design der Studie TEDS-LT. In S. Blömeke, A. Bremerich-Vos, G. Kaiser, G. Nold, H. Haudeck, J.-U. Keßler \& K. Schwippert (Hrsg.), Professionelle Kompetenzen im Studienverlauf. Weitere Ergebnisse zur Deutsch-, Englischund Mathematiklehrerausbildung aus TEDS-LT (S. 25-46). Münster: Waxmann.

Tatto, M. T., Schwille, J., Senk, S., Ingvarson, L., Rowley, G., Peck, R., Bankov, K., Rodriguez, M., \& Reckase, M. (2012). Policy, practice, and readiness to teach primary and secondary mathematics in 17 countries. Findings from the IEA teacher education and development study in mathematics (TEDS-M). http://files.eric.ed.gov/fulltext/ED542380.pdf. Zugegriffen: 12. Juni 2017.

Terhart, E. (1993). Pädagogisches Wissen. Überlegungen zu seiner Vielfalt, Funktion und sprachlichen Form am Beispiel des Lehrereissens. In J. Oelkers \& H.E. Tenorth (Hrsg.), Pädagogisches Wissen (S. 129-141). Weinheim: Beltz.

Terhart, E. (2012). „Bildungswissenschaften“. Verlegenheitslösung, Sammeldisziplin, Kampfbegriff? Zeitschrift für Pädagogik, 58(1), 22-39.

Van Driel, J.H., Verloop, N., \& de Vos, W. (1998). Developing science teachers' pedagogical content knowledge. Journal of research in Science Teaching, 35(6), 673-695.

Voss, T., Kunter, M., \& Baumert, J. (2011). Assessing teacher candidates' general pedagogical/psychological knowledge: Test construction and validation. Journal of Educational Psychology, 103(4), 952-969.

Voss, T., Kunina-Habenicht, O., Hoehne, V., \& Kunter, M. (2015). Stichwort Pädagogisches Wissen von Lehrkräften: Empirische Zugänge und Befunde. Zeitschrift für Erziehungswissenschaft, 18(2), 187-223.

Watson, C., Seifert, A., \& König, J. (2012). Institutionalisierung der erziehungswissenschaftlichen Lehrerausbildung: Charakterisierung der einbezogenen Standorte und Ausbildungsgänge. In J. König \& A. Seifert (Hrsg.), Lehramtsstudierende erwerben pädagogisches Professionswissen. Ergebnisse der Längsschnittstudie LEK zur Wirksamkeit der erziehungswissenschaftlichen Lehrerausbildung (S. 44-86). Münster: Waxmann.

Weber, G. (1997). Top languages: The world's 10 most influential languages. Language Today, 2.

Weinert, F.E. (1994). Lernen lernen und das eigene Verstehen lernen. In K. Reusser \& M. Reusser-Weyeneth (Hrsg.), Verstehen. Psychologischer Prozess und didaktische Aufgabe (S. 183-205). Bern: Huber. 
Wirtz, M.A., \& Caspar, F. (2002). Beurteilerübereinstimmung und Beurteilerreliabilität: Methoden zur Bestimmung und Verbesserung der Zuverlässigkeit von Einschätzungen mittels Kategoriensystemen und Ratingskalen. Göttingen: Hogrefe.

$\mathrm{Wu}, \mathrm{M}$. L. (1997). The developement and application of a fit test for use with generalised item response model (Unveröffentlichte Publikation). Melbourne, Australia: University of Melbourne. 